\title{
A theory of esteem based peer pressure*
}

\author{
Fabrizio Adriani $^{\dagger} \quad$ Silvia Sonderegger ${ }^{\ddagger}$
}

\begin{abstract}
How does the incentive to engage in social signaling depend on the composition of peers? We find that an increase in the mean peer quality may either strengthen signaling incentives (keeping up with the Joneses) or weaken them (small fish in a big pond). Both right and left truncations of the distribution of peer quality reduce signaling incentives, while more dispersed peer distributions strengthen them. Finally, more right skewed peer distributions strengthen signaling incentives when only a small fraction of the group engage in signaling, but weaken them when signaling is widespread.
\end{abstract}

JEL Codes: D82

Keywords: Esteem, Status, Peer pressure, Signaling, Small fish in a big pond, Conspicuous consumption, Distributional comparative statics.

\footnotetext{
*We thank an associate editor and two anonymous reviewers for their constructive comments which have helped to improve the paper. We are also indebted to Roland Bénabou, Subir Bose, Martin Jensen, Chris Wallace, Eyal Winter and Piercarlo Zanchettin for comments given at various stages of the project.

${ }^{\dagger}$ University of Leicester. University Road, Leicester, UK, LE1 7RH; e-mail: fa148@le.ac.uk.

${ }^{\ddagger}$ University of Nottingham and CeDEx. Sir Clive Granger Bld, University Park, Nottingham, UK, NG7 2RD; e-mail: silvia.sonderegger@nottingham.ac.uk.
} 


\section{Introduction}

More than thirty years ago, John Harsanyi noted that "apart from economic payoffs, social status seems to be the most important incentive and motivating force of social behavior." (Harsanyi, 1980). At least since then, economists have tried to incorporate the incentives arising from status into their formal models. A branch of the literature uses signaling theory to capture notions of status and esteem concerns 11 In these models, each agent is characterized by an unobservable attribute (ability, wealth, altruism) and is concerned about society's perception of his attribute. When he decides to adopt or not a particular behavior (study towards a degree, buy an expensive car, donate to charity), he takes into account the gain or loss in esteem - measured by the change in society's perception - associated with that behavior. This literature has proved successful in explaining a range of empirical phenomena (see Ellingsen and Johanneson, 2007). However, little attention has been devoted to understanding if and how the characteristics of one's peers affect the incentives to engage in costly signaling 2 This is what we wish to do in this paper.

The underlying idea is very simple. Belonging to a particular group (school, firm, fraternity, sport club, academic institution) conveys information about one's own characteristics (wealth, ability, social skills). This is because different groups typically carry different distributions of relevant attributes among their members. For instance, job market candidates from prestigious academic institutions are automatically accorded higher expected ability than candidates from second tier institutions. As noted by Charles et al. (2009), this opens the door to the possibility that identical individuals who happen to belong to different groups may face different pressure to engage in status-enhancing signaling.

\footnotetext{
${ }^{1}$ Examples include Bernheim (1994), Ireland, (1994), Glazer and Konrad (1996), Corneo and Gruener (2000), Bénabou and Tirole (2006 and 2011), Ellingsen and Johanneson (2008), Andreoni and Bernheim (2009), Harbaugh (2010).

${ }^{2}$ Economic theories of peer effects are based on complementarities in production (see e.g. Arnott and Rowse, 1987, Bénabou, 1993, Lazear, 2001), and on information externalities (Banerjee and Beasley, 1991). There is also work that looks at peer pressure and peer monitoring in team production (Kandel and Lazear, 1992, Barron and Gjerde, 1997). Another strand of the literature - see e.g., Adriani and Sonderegger (2009, 2018) and Adriani et al. (2018) - builds on the idea that, through parental socialization choices à la Bisin and Verdier (2001), the distribution of traits in the population and peer pressure to conform to social norms are jointly determined. None of these elements is present in our model.
} 
In this paper, we look at this problem from the perspective of the "honor-stigma" model proposed by Bénabou and Tirole $(2006,2011)$. This model is extremely simple and yet, we argue, generates a rich set of predictions. The primary reason for the model's simplicity is that, differently from other signaling models of status, agents can only signal their attributes by performing or not a discrete task. The discrete nature of the action space implies that information conveyed by one's behavior is coarse, ensuring that some degree of pooling always occurs in equilibrium. This provides a natural channel for the shape of the peer group distribution to affect signaling incentives and differentiates the setting from environments with continuous actions, where full separation is possible ${ }^{3}$ Another advantage is that these non-convexities map salient features of real world problems in a compelling way. For instance, educational attainments are typically discrete in nature and are marked by events (e.g. graduation ceremonies) that emphasize their discreteness. Similarly, status symbols (luxury watches, bespoke suits, sports cars) are typically coarse signals of one's wealth. Indeed, it is precisely their potential as "pooling devices" that makes these goods attractive to many.

We add to existing literature by providing a systematic analysis of the comparative statics of the "honor-stigma" model, which enables us to derive novel results. We use the model to ask the following questions: What is the effect of belonging to a "better" (smarter, richer, more pro-social) group on the incentive to engage in costly signaling? What is the effect of a more diverse group? What is the effect of segregation by ability (e.g. admissions policies to academic programmes)? What happens when the upper tail self selects out of the group?

Our first result focuses on how the signaling incentives are affected by a shift in the mean quality of the group. Consider for instance the Boston Metropolitan Council for Educational Opportunity (Metco) program, that sends students from Boston schools to more affluent suburbs (where average ability is typically higher). What is the effect of this kind of displacement on a pupil's incentives to signal his ability? Bénabou and Tirole (2006) point out that behavior in the "honor-stigma" model can display strategic complementarity or substitutability. We show that this naturally translates into two possible scenarios, a "keeping up with the Joneses" effect, where better peers increase one's incentive to perform,

\footnotetext{
${ }^{3}$ Optimal signal design often leads to coarse signals, see Harbaugh and Rasmussen (2014). In the case of grading structures, Dubey and Geanakoplos (2010) show that a degree of coarseness is optimal when students play status games (see also Zubrickas, 2015). Mazali and Rodrigues-Neto (2013) find that it is optimal for status good producers to offer a finite number of brands/varieties.
} 
or a "small fish in a big pond" effect, where better peers may actually lower motivation 4 We characterize the conditions under which the pressure to provide the costly signal increases or decreases in peer quality. We show that these crucially depend on the shape and location of the peer distribution. With symmetric unimodal distributions, an individual moving to a higher quality group will face stronger incentives when the costly signal is provided by the majority in both groups. However, if the signal is rare, then incentives are unambiguously weakened (the small fish in a big pond effect). In that case, to boost incentives, someone would actually have to be moved in the opposite direction, from the high to the low quality group. This is because, in the low quality group, the costly signal is even rarer, and hence the esteem ('honor') associated with signaling is stronger.

We then move to the case of a U-shaped distribution of types, where extreme types are the most frequent. This is is for instance the case when considering household income in areas with high wealth inequality, where the middle class is practically non-existent. 5 In fact, this type of distribution can be seen as a stylized version of a "polarized" or bimodal group. With a U-shaped distribution we obtain opposite predictions to the unimodal case. For instance, the small fish in a big pond effect is obtained when the costly signal is majoritarian in both groups. As it will become clear, the reason for the reversal in the direction of comparative statics is that, with unimodal distributions, incentives are strongest when the costly signal is either provided by nearly everyone or almost no one. With U-shaped distributions, the opposite happens. Incentives are strongest when the group is evenly split between those who provide the signal and those who don't.

Having looked at a shift in peer quality, we turn to peer heterogeneity. First, we assess the effect of imposing truncations on the group distribution. This has clear policy relevance. For instance, admission policies based on academic ability have been used in many countries to establish selective schools where the distribution of pupils' ability is left-truncated ${ }^{6}$ In a different context, left censored income distributions may be generated by policies mandating

\footnotetext{
${ }^{4}$ The small fish in a big pond effect is the subject of a sizeable empirical literature in Psychology (see Marsh, 1987, and subsequent work), but has received little attention in theoretical Economics. A notable exception, in a quite different context, is Frank (1985a).

${ }^{5} \mathrm{~A}$ case in point is the Washington D.C. area, where, according to 2014 data from the US Census Bureau, the two modal income brackets are incomes below $\$ 25,000$ per year and incomes above $\$ 150,000$. A U-shaped distribution is a good approximation for the distribution of household income.

${ }^{6}$ The old Grammar Schools system in Britain is a prominent example.
} 
a guaranteed minimum income for all members of society, as recently proposed by several political commentators. We ask how these policies affect signaling incentives. Second, we analyze the effect of a mean preserving spread. This may help to address questions such as: How does the incentive to engage in conspicuous consumption change when inequality goes up? Finally, we look at the effect of skewness, restricting attention to pairs of distributions with identical mean that cannot be ranked in terms of second order stochastic dominance. This may shed light, for instance, on what happens to the propensity to purchase positional goods when the top $1 \%$ of the income distribution pull away from everyone else.

All our results are linked by a common theme: fatter tails increase signaling incentives. Whenever distributions can be clearly ranked according to their tails, this delivers unambiguous predictions. This is for instance the case for truncations, where the two distributions are isomorphic except for the fact that one (the truncated distribution) has the right or left tail missing. The model accordingly predicts that the original distribution (the one without truncation) provides stronger signaling incentives. This happens irrespectively of whether the truncation is on the lower tail or on the upper tail. Intuitively, right truncations reduce the honor from providing the costly signal, while left truncations reduce the stigma associated with failing to provide it.

In the case of second-order stochastic dominance the comparison between peer distributions is similarly unequivocal. In a more dispersed group, engaging in costly signaling means pooling with a fatter tail of extremely high types and separating from a fatter tail of extremely low types. Both effects go in the same direction of increasing the value of the signal and thus increasing incentives. Interestingly, this result stands in sharp contrast with the predictions of recent literature on rank based comparisons (see e.g. Hopkins and Kornienko, 2009). We discuss in the next section why the two approaches deliver opposite predictions.

Finally, when considering skewness, we must rely on conditional predictions. The reason is quite transparent. If $f$ is more right-skewed than $f^{\prime}$, this implies that $f$ has a fatter right tail but a thinner left one. It is thus not possible to "rank" the two distributions based on how fat their tails are. Nonetheless, the logic we have highlighted persists in those cases where one tail is clearly more important than the other for incentive provision. Consider for instance environments where the incentive to undertake costly signaling is primarily motivated by honor (obtained by showing that one belongs to a handful of high type individuals providing 
the signal). In those cases, intuition suggests that a fatter right tail may be better suited to motivate agents. Accordingly, we find that a more right skewed distribution provides stronger incentives. The opposite happens in environments where, in equilibrium, incentives are primarily motivated by stigma avoidance. In these cases, incentives are stronger with a more left skewed distribution.

The main focus of our analysis is to characterize how the signaling incentives for a given individual are affected by different peer type-distributions. Empirically, this is relevant when it is possible to control for an individual's type (e.g. through a within-subject type of analysis). In some cases, however, within-subject data may not be available, but it may still be possible to measure the aggregate effect of a policy change or an economic shock. It is therefore instructive to investigate how aggregate signal provision changes under different type distributions. For instance, if $f^{\prime}$ is a mean preserving spread of $f$, a relevant question may be: how does the proportion of those providing the signal (e.g., engaging in conspicuous consumption) in $f$ compare with that in $f^{\prime}$ ? From this viewpoint, the change from $f$ to $f^{\prime}$ does not only affect incentives, but also the frequency of each type, thus generating a composition effect. Intuitively, a mean preserving spread of the income distribution may increase the number of agents who are too poor to afford the signal (as well as the super rich). As a result, the effect of higher dispersion on aggregate provision is not a priori obvious in spite of the fact that peer pressure is unambiguously stronger under $f^{\prime}$. In the paper, we provide a number of results that shed light on the interplay between incentive and composition effects. In particular, we ask when these effects go in the same direction, and when they counteract each other.

It is worth mentioning that we use the expression incentives without any positive or negative connotation. The welfare consequences of stronger incentives typically depend on the precise application. For instance, incentives are presumably good when talking about educational attainments, but are harmful when considering wasteful "money burning" types of activities. Indeed, our running application and the related welfare analysis will focus on the case of socially wasteful signals. As we will see, in these cases heavier tails are not necessarily beneficial for society. 


\subsection{Outline of the paper and relationship with the literature}

This paper builds on and brings together a number of existing results, some of which will need to be recast for completeness. Sections 2 and 3 review the "honor-stigma model" following Bénabou and Tirole (2006) and extend it to peer groups with U-shaped distributions. Clearly enough, although our results are cast in terms of esteem concerns, the analysis applies equally well to any binary action/continuous type version of Spence's (1973) model - where the sender's utility is increasing in the receiver's posterior belief. While Bénabou and Tirole $(2006,2011)$ mostly focus on the effects of monetary incentives and information disclosures, Jewitt (2004) provides a number of technical results on the shape of distributions, pointing to the "honor-stigma" model as a potential application 7 What we add to this literature is, first of all, a systematic analysis of the distributional comparative statics (DCS) of signaling incentives.

DCS on individual incentives In Section 4 we look at how the incentives to provide the signal for each type of agent are affected by changes in the distribution of their peers' quality. In practice, this reduces to studying how the marginal type - who is indifferent between providing the signal and abstaining - responds to changes in the peer distribution. Our first result considers the effect of a uniform shift in the quality distribution of peers. Bénabou and Tirole (2011) also look at the implications of this, but mostly within the context of aggregate provision. We extend their results in the following directions: a) We determine how (and when) individual incentives may actually decrease as peer quality improves; b) We point out that the DCS dramatically change if the shape of the peer distribution changes from unimodal to bimodal - as is for instance when comparing two neighborhoods where most people are middle class or two neighborhoods where the middle class is absent. We then move beyond uniform shifts to study the effects on incentives of changes in dispersion, changes in skewness and of truncations of the peer distribution. The analysis builds on a number of observations made by Jewitt (2004) - most notably, that the gains in esteem from providing the signal can be represented in terms of the Lorenz curve associated with the peer distribution. However, the comparative statics results we derive are, to our knowledge,

\footnotetext{
${ }^{7}$ Some of Jewitt's results are not in the public domain. We thus provide proofs for some of his results that, to our knowledge, have not already been proved elsewhere. Any inaccuracy in any of these proofs should be attributed exclusively to us.
} 
entirely novel. As mentioned, the main theme that emerges from the analysis is that, in general, "fatter tails" strengthen incentives.

DCS on aggregate provision A second contribution of this paper to the literature on esteem concerns is that, even within the context of aggregate effects, we are able to extend the analysis of Bénabou and Tirole by looking at a number of manipulations of the peer distribution, beyond simple uniform shifts. Section 5 provides comparative static results on aggregate signal provision. In particular, we derive sufficient conditions for the proportion of agents providing the signal to be increasing in dispersion, which links our work to the literature on the effect of inequality on status seeking behavior. Our analysis differs from most existing works on this topic in a number of respects. First, when looking at aggregate provision, we find that dispersion both (i) affects incentives and thus the marginal type (incentive effect); (ii) affects the frequency of each type, so that, even keeping incentives constant, the proportion of agents providing the signal changes (composition effect). The literature mostly focuses on fully separating outcomes (see e.g. Glazer and Konrad, 1996). Under perfect separation, a change in the shape of the distribution only has a composition effect but virtually no incentive effect ${ }^{8}$ In our case, both effects are present. Second, different from most of the literature, the binary nature of agents' problem implies that the mapping from types to optimal actions is a step function. Hence, standard techniques (e.g. Atkinson, 1970, Jensen, 2018) based on the concavity/convexity of the policy function are not directly applicable.

Welfare and redistribution In Section 6, we apply our results to analyze welfare and redistribution when signaling is purely wasteful and takes the form of conspicuous consumption. Our focus is on a particular DCS, namely a compression in the income distribution generated by higher taxes and transfers. Although intuition would suggest that more redistribution always reduces signal provision, we find that this is not necessarily the case. In particular, for low levels of redistributions, the relatively disadvantaged may choose to spend the extra income on conspicuous consumption to separate from the very poor. Nonetheless, we show that signal provision always decreases when redistribution is high enough. This result echoes some of the effects highlighted in Levy and Razin (2015). Using a slightly

\footnotetext{
${ }^{8}$ This is because, in a separating equilibrium, incentive compatibility constraints do not depend on the frequency of each type. As a result, incentives may be affected only by changes in the distribution's support.
} 
different setting, they look at how costly income sorting into exclusive "clubs" may affect preferences for redistribution. In line with their analysis, we also provide a number of results on how the presence of income signaling affects the support for redistribution in society and discuss the main differences between the two approaches.

Rank based comparisons The finding that an increase in dispersion may increase signal provision is in contrast with the literature on rank-based comparisons, which typically finds that higher inequality reduces the incentive to engage in status seeking behavior (see Hopkins and Kornienko, 2004 and 2009) 9 These models differ from ours both in the different way status is attained - through rank rather than through beliefs about one's type - and in the nature of the action space (continuous rather than binary). As discussed in Section 8, in a binary action framework where agents care about perceived rank, rather than perceived type, the social incentive to act does not depend on the distribution of types 10 In Hopkins and Kornienko's work, however, actions are continuous. This ensures that the distribution of types matters for incentives in spite of rank-based concerns. In their setup, higher inequality reduces social competition because it increases the cost of keeping up with people higher up on the social ladder. In contrast, in the honor-stigma framework, higher inequality increases the esteem rewards from being pooled with the upper tail, thus providing stronger incentives. Intuitively, these different predictions arise because changing the wealth distribution in the honor-stigma model affects both individual endowments and the rewards from signaling, while in a rank-based framework it only affects the distribution of endowments (see Hopkins and Kornienko, 2010).

Finally, in Section 7 we briefly look at robustness to endogenous group formation. Section 8 offers concluding remarks.

\footnotetext{
${ }^{9}$ These models build on the work of Frank (1985b). Weiss and Fershtman (1998) and Postlewaite (2011) provide surveys of status models in Economics.

${ }^{10}$ This is because rank is always uniformly distributed. As a result, if only agents with type above $x$ provide the signal, the difference in perceived rank between someone providing the signal and someone abstaining is always the same independently of the underlying distribution. See also Section 8 .
} 


\section{The honor-stigma model}

Each agent belongs to a group $g$ (this could be an ethnic/cultural group, but also a school, firm, fraternity, sport club). A group $g$ has a continuum of (heterogenous) members indexed by their type $x \in\left[\underline{X}_{g}, \bar{X}_{g}\right]$. The density of $x, f_{g}:\left[\underline{X}_{g}, \bar{X}_{g}\right] \rightarrow \mathbb{R}^{+}$, is assumed to be twice differentiable, to have full support, a strictly increasing cumulative $(\mathrm{CDF}) F_{g}$, a quantile function $F_{g}^{-1}$, and a finite mean $\theta_{g}$. All group members simultaneously choose action $a \in$ $\{0,1\}$. Group membership and the decision to provide the signal $(a=1)$ or abstain $(a=0)$ are publicly observable but a member's type $x$ is not.

Each group member has preferences which depend on material welfare and esteem. Following Bénabou and Tirole, a type $x$ individual has utility,

$$
U(a, x)+E(x \mid a, g) .
$$

The first term captures the intrinsic utility associated to performing the action. The function $U:\{0,1\} \times \mathbb{R}^{+} \rightarrow \mathbb{R}^{+}$is assumed to be twice differentiable in $x$ and with increasing differences - i.e. $v(x) \equiv U(1, x)-U(0, x)$ is strictly increasing - implying that performing the action is less costly for (or affords higher benefits to) higher types. The second term reflects the utility that a group member derives from esteem. The interpretation is that the group member is concerned about the assessment of his type made by an external audience. The external audience could be fellow group members or some individual (e.g. a potential mate or employer) outside the group.

\section{Preliminaries}

We first characterize the equilibrium taking the distribution of types for group $g$ as given. A perfect Bayesian equilibrium is a profile of strategies for group members and a belief function for the audience such that: i) the strategy of each member is a best reply to the strategies of others and the audience's beliefs, ii) beliefs are derived from members' strategies using Bayes rule where possible. We say that the decision to perform the action is non-trivial for group $g$ if

$$
v\left(\bar{X}_{g}\right)+\bar{X}_{g}-\theta_{g}>0>v\left(\underline{X}_{g}\right)+\theta_{g}-\underline{X}_{g} .
$$


where, as already mentioned, $v(x) \equiv U(1, x)-U(0, x)$. In words, it is strictly optimal for the highest type to choose $a=1$ when others choose $a=0$ if, by doing this, he can reveal himself. Symmetrically, it is strictly optimal for the lowest type to choose $a=0$ even if this reveals that he is the lowest type. In the remainder of the paper, whenever we will compare two groups, we will assume that this condition is satisfied for both groups.

If the decision to perform the action is non-trivial, then, by continuity, there is at least one equilibrium with an interior cutoff $x^{*}$, i.e. such that any member performs if and only if $x \geq x^{*} 11$ The marginal type $x^{*}$ is implicitly given by the indifference condition,

$$
\phi_{g}\left(x^{*}\right)=-v\left(x^{*}\right)
$$

where

$$
\begin{aligned}
\phi_{g}(x) & \equiv m^{+}(x ; g)-m^{-}(x ; g), \\
m^{+}(x ; g) & \equiv E(X \mid X>x ; g), m^{-}(x ; g) \equiv E(X \mid X<x ; g)
\end{aligned}
$$

The function $\phi_{g}$ captures the gain in esteem obtained by providing the signal under a strategy profile such that only types above $x$ provide it. The value taken by $x^{*}$ can be seen as an inverse measure of the strength of the incentives generated by peer pressure. When $x^{*}$ decreases, all types previously providing the signal keep sending it, while some types who were previously abstaining switch to signal provision.

The shape of $\phi_{g}$ determines how incentives change with different cutoff values. Suppose for instance that $\phi_{g}$ is negatively-sloped. This implies that, as provision becomes more common (i.e., the cutoff decreases), the pool of abstainers worsens more than that of signal providers. As a result, the esteem gain associated with signal provision increases (or, equivalently, the stigma associated with abstention increases), thus strengthening signaling incentives. In other words, we have (endogenous) strategic complementarities. By converse, when $\phi_{g}$ is positively sloped, wider signal provision worsens the pool of abstainers less than it worsens that of signal providers. The honor associated with signaling is thus reduced, generating (endogenous) substitutability. Clearly enough, the shape of $\phi_{g}$ (and thus the nature of the strategic interaction) is determined by the shape of the underlying density $f_{g}$. In some cases, we will restrict attention to densities which experience at most one change of

\footnotetext{
${ }^{11}$ Full pooling equilibria where all agents abstain may also exist. However, given monotonicity of $v$, these equilibria fail standard refinements like D1 (see Cho and Kreps, 1987). See supplementary material.
} 
monotonicity. This includes the cases familiar in the literature (Jewitt, 2004) of increasing, decreasing, and unimodal densities, as well as the less familiar case of U-shaped densities.

Lemma 1. a) (Jewitt, 2004) If, $f_{g}$ is everywhere increasing (decreasing), then $\phi_{g}$ is everywhere decreasing (increasing). b) (Jewitt, 2004) If $f_{g}$ is increasing and then decreasing (unimodal), then $\phi_{g}$ is quasi-convex. c) If $f_{g}$ is decreasing and then increasing (U-shaped), then $\phi_{g}$ is quasi-concave.

Proof. See Jewitt (2004) (or Harbaugh and Rasmussen, 2014) for a) and b). We prove c) in the Appendix using an argument mirroring Harbaugh and Rasmussen (2014).

The quasi-convexity/concavity results in Lemma 1 do not tell us much about the relationship between the esteem gain $\phi_{g}$ and $x$. These results can be strengthened if we focus on densities such that, for all values of $x$ in the support except (possibly) the one where monotonicity changes, are either strictly increasing or decreasing. [In the rest of the paper, we will slightly abuse terminology using the expression "with at most one change of monotonicity" to indicate this type of densities.]

Lemma 2. (Unimodal case) If $f_{g}$ is strictly increasing and then decreasing, then there exists $x_{\text {min }} \in\left[\underline{X}_{g}, \bar{X}_{g}\right]$ such that $\phi_{g}$ is strictly decreasing for $x<x_{\text {min }}$ and strictly increasing for $x>x_{\text {min }}$. If $f_{g}$ is symmetric, then $x_{\text {min }}$ coincides with the mode of $f_{g}$.

(U-shaped case) If $f_{g}$ is strictly decreasing and then increasing, then there exists $x_{\max } \in$ $\left[\underline{X}_{g}, \bar{X}_{g}\right]$ such that $\phi_{g}$ is strictly increasing for $x<x_{\max }$ and strictly decreasing for $x>x_{\max }$. If $f_{g}$ is symmetric, then $x_{\max }$ coincides with the anti-mode of $f_{g}$.

Proof. See appendix.

Note that, $x_{\min }$ and $x_{\max }$ need not lie in the interior of the support (although this always happens in the case of symmetric distributions). Harbaugh and Rasmussen (2014) provide conditions under which $x_{\min }$ is interior. These can be interpreted as requiring that $f_{g}$ is not too skewed. Similar results can be obtained for the U-shaped case.

The relationship between esteem concerns (captured by $\phi_{g}$ ) and the shape of $f_{g}$ is illustrated in Figure 1 for symmetric densities.

Consider first the case where $f_{g}$ is unimodal (drawn on the figure's left panel). Here, esteem concerns are higher (i.e., $\phi_{g}(x)$ is larger) when the marginal type is located at the 

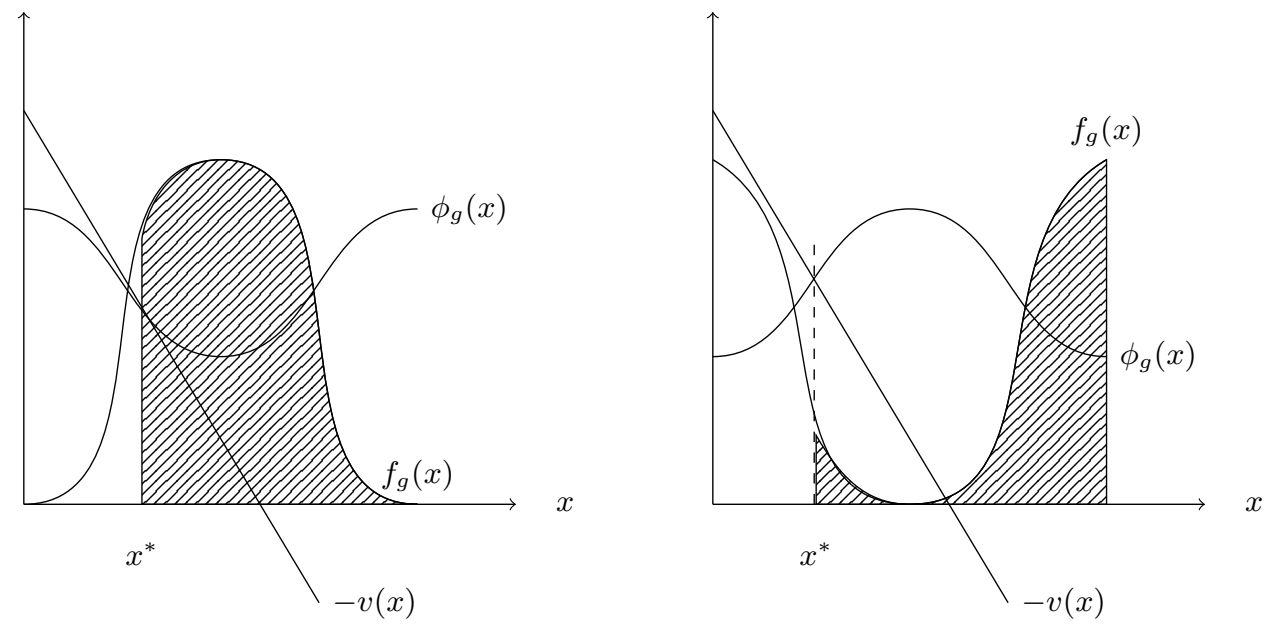

Figure 1: Equilibrium with unimodal and U shaped symmetric densities.

extremes of the distribution. This happens when the group is divided very unevenly between signal provision and abstention, i.e. the signal is either provided by a large majority or by a small minority. Intuitively, in these cases people are strongly motivated by the desire to separate themselves from a few "bad apples", or by the desire to establish themselves as part of a selected minority of "stars". When $f_{g}$ is U-shaped, the opposite happens. Esteem concerns are highest when the marginal type is intermediate. This happens when the group is evenly split between provision and abstention. Intuitively, this is because, when the distribution is U-shaped, an intermediate cutoff makes the act of performing the action very informative about the individual's type.

From Lemma 2, the LHS in (3) is a non-monotonic function while the RHS is strictly decreasing. A sufficient condition for a unique interior cutoff is thus that the RHS is everywhere steeper than the LHS. Following Bénabou and Tirole (2006, 2011), we will accordingly assume that, for all groups involved,

Assumption 1. (Uniqueness) $-v^{\prime}(x)<\phi_{g}^{\prime}(x)$ for all $x \in\left[\underline{X}_{g}, \bar{X}_{g}\right]$.

In the supplementary Appendix, we do away with this assumption and show that results similar in spirit to the results discussed here can be obtained by looking at extreme equilibria (see e.g. Milgrom and Roberts, 1994). These are the equilibria with the lowest and highest provision. 


\section{DCS and individual incentives}

In this section, we look at how incentives change as a result of manipulations of the peer distribution - as captured by the DCS on the marginal type $x^{*}$.

\subsection{Uniform shifts}

In order to isolate the effect of a change in group quality on individual incentives, we consider a family of identical densities which differ only in the mean parameter $\theta_{g}$. It is clear that such uniform shifts of the density $f_{g}$ will shift the net esteem function $\phi_{g}$ in the same way.

Proposition 1. Consider two groups $g$ and $g^{\prime}$ with $f_{g}$ and $f_{g^{\prime}}$ differing only in their mean, with $\theta_{g}<\theta_{g^{\prime}}$, and having at most one change of monotonicity. Then, $\phi_{g}$ and $\phi_{g^{\prime}}$ cross at most once in $\left(\underline{X}_{g^{\prime}}, \bar{X}_{g}\right)$. When the densities are non-monotone and $\phi_{g}$ and $\phi_{g^{\prime}}$ cross:

$i x_{g}^{*}$ and $x_{g^{\prime}}^{*}$ are always either both on the left or both on the right of the crossing point $\hat{x}$.

ii Unimodal case: If both $x_{g}^{*}$ and $x_{g^{\prime}}^{*}$ are on the right (left) of $\hat{x}$, then $x_{g}^{*}<(>) x_{g^{\prime}}^{*}$.

iii $U$-shaped case: If both $x_{g}^{*}$ and $x_{g^{\prime}}^{*}$ are on the right (left) of $\hat{x}$, then $x_{g}^{*}>(<) x_{g^{\prime}}^{*}$.

Moreover, if $f_{g}$ and $f_{g^{\prime}}$ are symmetric, then $\phi_{g}$ and $\phi_{g^{\prime}}$ necessarily cross and the crossing point lies in the interval $\left(\theta_{g}, \theta_{g^{\prime}}\right)$. Finally, when the densities are monotone (or are nonmonotone but $\phi_{g}$ and $\phi_{g^{\prime}}$ do not cross), then $x_{g^{\prime}}^{*}<(>) x_{g}^{*}$ whenever $\theta_{g}>(<)\left(\bar{X}_{g}+\underline{X}_{g}\right) / 2$.

Proof. See appendix.

The implications of Proposition 1 can be easily grasped by focusing on the case of symmetric densities. Figures 2 and 3 illustrate the effect of a shift in the density on the equilibrium cutoff for symmetric unimodal and U-shaped densities, respectively. Consider first the unimodal case in Figure 2. In this case, esteem concerns are strongest when provision is either extremely high or extremely low. Suppose we take a group $g$ in which signal provision is majoritarian, i.e., $x_{g}^{*}<\theta_{g}$, and we compare it with a higher quality group $g^{\prime}$, as in the left panel of Figure 2, Clearly enough, evaluated at $x=x_{g}^{*}$, the higher quality group will exhibit more signal provision (since a larger share of agents are located above $x$ ). Hence, in the high quality group, the majoritarian behavior (i.e., providing the signal) will be even more common. Incentives will therefore be stronger. The marginal type for the high quality group 

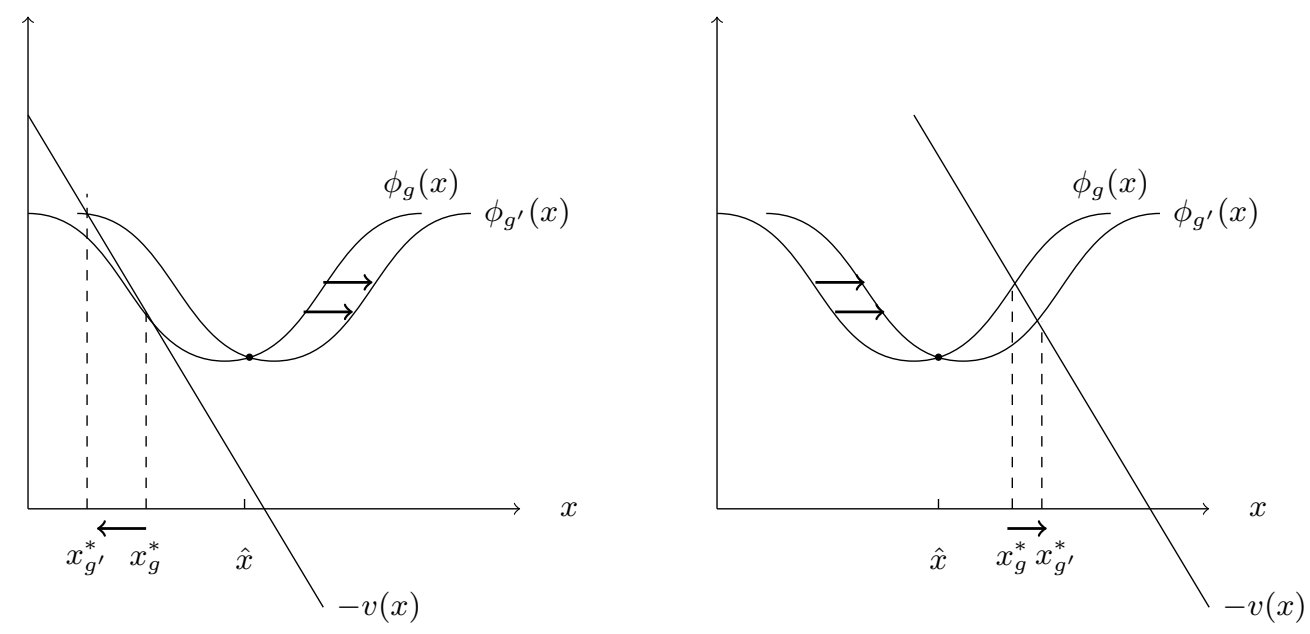

Figure 2: Effect of better peers when action provision is majoritarian (left) and minoritarian (right) [Unimodal density].
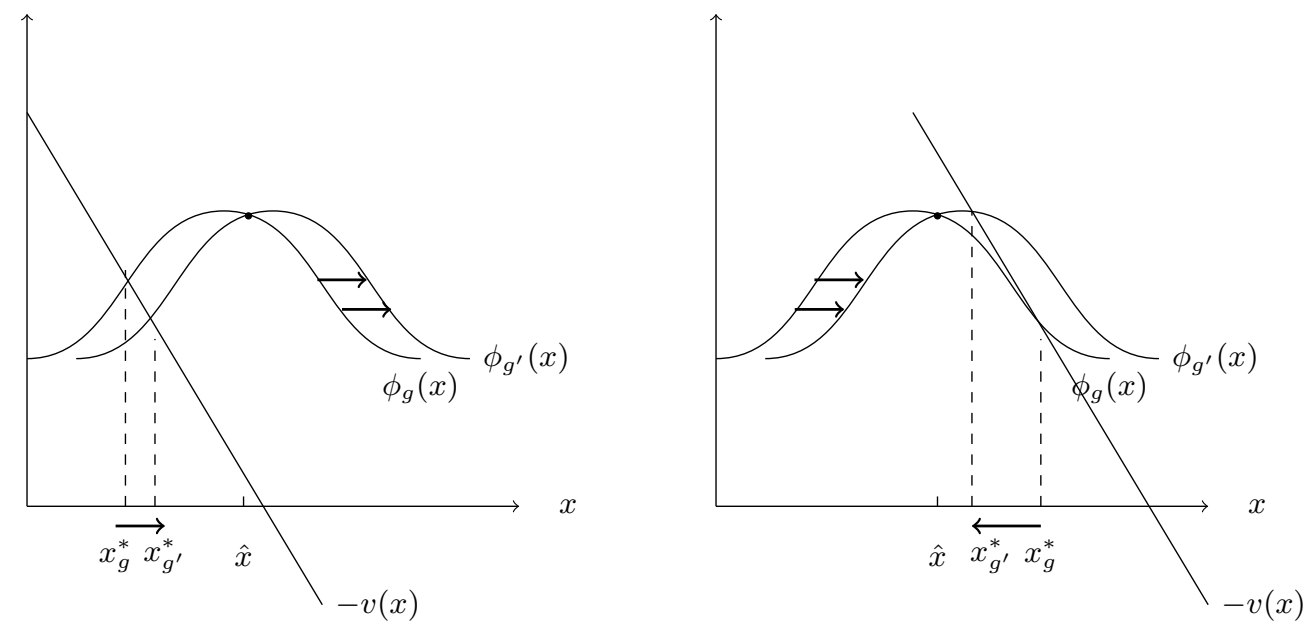

Figure 3: Effect of better peers when action provision is majoritarian (left) and minoritarian (right) [U-shaped density]. 
must therefore lie below that of the low quality group. This has very practical implications. If an individual moves from a group in which provision is majoritarian to a higher quality group, he faces stronger incentives: the keeping up with the Joneses effect.

Consider now a group in which provision is minoritarian, i.e., $x_{g}^{*}>\theta_{g}$ (right panel of Figure 22. Evaluated at $x=x_{g}^{*}$, a higher quality group $g^{\prime}$ will exhibit more signal provision (since a larger share of members are located above $x$ ). In the lower quality group $g$, the majoritarian behavior (i.e., abstention) will thus be more common, and, hence, esteem concerns will be stronger. The marginal type for the lower quality group must therefore lie below that of the higher quality group. This delivers an intriguing implication. If an individual moves from a group in which signal provision is minoritarian to a higher ability group, he faces weaker incentives: the small fish in a big pond effect.

What about U-shaped densities? The mechanism at work is exactly the mirror image: esteem concerns are stronger in the group that is closer to a half-half split. As Figure 3 shows, this generates opposite comparative statics to the unimodal case. To sum up, Proposition 1 delivers the following rule of thumb, $\sqrt{12}$

Corollary 1. In the symmetric unimodal (resp., U-shaped) case, if an individual is moved - from a group in which provision is majoritarian to a higher quality group, or - from a group in which provision is minoritarian to a lower quality group, he faces stronger (resp., weaker) incentives to provide the signal.

Finally, note that similar effects obtain with asymmetric densities. However, in that case, whether the $\phi$ functions are increasing or decreasing will not perfectly map majoritarian/minoritarian behavior. Moreover, if the densities are very skewed (as for instance in the case of monotone densities) it may happen that the $\phi$ functions never cross. In this case, one of the two groups will provide uniformly stronger incentives. Nevertheless, the fundamental insights are unchanged even in this case. For instance, if the density is strongly left skewed (e.g. a strictly increasing density), the $\phi$ function will be generally downward sloping. In

\footnotetext{
${ }^{12}$ Notice however that Proposition 1 delivers a more general result, which allows to say what happens also when one moves from a group where provision is minoritarian to a better group or from a group with majoritarian provision to a worse group. In these cases, the direction of the effect crucially depends on whether the two cutoffs $x_{g}^{*}$ and $x_{g^{\prime}}^{*}$ lie on the left or on the right of the point $\hat{x}$ where the esteem gain from signal provision is equal in both groups.
} 
other words, the gain in esteem tends to fade as more agents provide the signal. In turn, this implies that the higher quality group - which has higher provision - will provide uniformly weaker incentives. Symmetrically, with a strongly right skewed density (e.g. a decreasing density), the gain in esteem increases as more agents choose to provide the signal, so that the higher quality group provides stronger incentives.

The small fish in a big pond effect One of the key lessons of Proposition 1 is that better peers may in some cases discourage, rather than encourage costly signaling ${ }^{13}$ In this case, moving the individual to a higher quality group may actually reduce motivation. With unimodal densities, this small fish in a big pond effect emerges when signal provision is rare, like for instance in the case of the award of a special prize. Winning such a prize may be very valuable to a bright pupil from a school full of disadvantaged kids, since it allows him/her to separate from the rest of the student body - which is perceived as generally weak. The same may not be true if the same pupil attends a school mostly composed of middle class kids. By converse, with U-shaped densities, the small fish effect arises when most pupils are capable of sending the signal, like for instance passing a relatively easy test. Note however that, as Figures 2 and 3 suggest, the small fish effect is to some extent a local result. With unimodal densities, esteem incentives always increase if one moves to a group of sufficiently higher ability 14 Symmetrically, with U-shaped densities, incentives always decrease if one moves to a sufficiently worse group.Within the contest of education, the small fish in a big pond effect is extensively documented by social psychologists (see Marsh, 1987, and related literature). Psychological evidence suggests that pupils moving from mixed ability schools to better schools often feel demoralized (Marsh et al. 1995).

Finally, we ask what happens when the action $a$ is imperfectly observed by the audience.

\footnotetext{
${ }^{13}$ Given the binary nature of the signal, one may wonder what the relevant signal is. For instance, in the case of university graduates, is it whether or not a student graduates, or whether or not they receive an honors degree? The answer may depend on the relevant audience. For instance, official statistics tend to report the share of graduates in a given neighborhood, often without distinguishing between honors degrees and ordinary degrees. For some audiences (e.g. neighbors), there is probably not much loss of information in pooling honors degree and ordinary degree holders together. Of course, this may not be true if the audience is composed of potential employers, who may have a more direct incentive to distinguish between good graduates and mediocre ones.

${ }^{14}$ Note that this is nevertheless consistent with Proposition 1, since the shift would need to be large enough to move the crossing point $\hat{x}$ to the right of both cutoffs.
} 
This is analyzed in detail in the supplementary material. Two results stand out. First, a more noisy signal generally lowers esteem concerns. Second, the way noise is introduced is important. While in some cases all comparative statics trivially carry through, we provide a plausible example where additional effects arise. This is the case where the audience only observes "pass" or "fail" in a test, whose outcome is imperfectly correlated with the effort $a \in\{0,1\}$ exerted by the agent. In this case, we show that, as the test becomes more noisy, incentives are most weakened when the marginal type is extreme, so that provision is either very high or very low. Reducing the correlation between agents' action and observed signals is thus somehow similar to making the distribution more U-shaped. The intuition is that, when it is known that the overwhelming majority exert effort, a fail tends to be imputed to bad luck, thus carrying less stigma. For similar reasons, less honor is attached to a pass if it is known that hardly anyone exerts effort and the test has a degree of randomness.

\subsection{Truncations and censoring}

In many cases, group distributions may present some form of censoring or truncation, either left or right (or both). Examples of left truncations are admissions policies to academic programmes, where the objective is the exclusion of the less talented students. Right truncations are more rarely advertised but equally common. A typical example would be semiprofessional sport circuits, where the most talented have incentive to turn fully professional and thus leave the circuit. In this section we analyze the effect of truncations or censoring on the incentive to perform the status enhancing action. Note that a truncation affects both the group's mean and its heterogeneity. Different from the previous section, however, the change in the mean is not generated by a uniform shift in the distribution and the previous results are not immediately applicable. Suppose then that group $g^{\prime}$ is identical to group $g$, except for a left truncation of the distribution. Formally, for some $k$ in the support of $f_{g}$,

$$
f_{g^{\prime}}(x)=\left\{\begin{array}{cc}
\frac{f_{g}(x)}{1-F_{g}(k)} & x \geq k \\
0 & x<k
\end{array}\right.
$$

Right truncations are defined in a symmetric way. We also consider the case of censoring, whereby all the mass in the left (right) tail, $F(k)(1-F(k))$, is reallocated to an atom at point $k$. We retain the assumption that the decision to send the signal is non-trivial also for the truncated/censored group. In other words, we focus on truncations such that the mass 
of both abstainers and signal providers in the truncated/censored group remains strictly positive.

Proposition 2. Consider a group $g$ with density $f_{g}$ and let $g^{\prime}$ denote a group with density $f_{g^{\prime}}$ obtained by truncating or censoring $f_{g}$. Then, so long as $x_{g^{\prime}}^{*}$ is interior to the support of the truncated/censored distribution, the incentives to provide the signal under $g^{\prime}$ are weaker than under $g$, i.e. $x_{g^{\prime}}^{*}>x_{g}^{*}$. This applies irrespectively of whether the truncation/censoring is on the left or right tail (or on both tails).

Proof. See appendix.

Proposition 2 establishes a strong result: Truncations or censoring reduce incentives. This applies irrespectively of the shape of the original distribution. Intuitively, left truncations/censoring reduce the stigma associated with abstention, while right truncations/censoring reduce the honor associated with provision. Below, we provide an example of a policy leading to a left censoring of the distribution.

Example: Guaranteed minimum income and conspicuous consumption In recent years, several political commentators, both on the left and the right, have argued in favor of reforming the welfare systems of developed economies to guarantee a minimum income to all citizens/residents. Here, we analyze the effect of this policy on the propensity to engage in conspicuous consumption. Consider a consumer with material utility (i.e. abstracting from esteem) $U(a, C)$ where $C \geq 0$ is a composite good and $a \in\{0,1\}$ denotes whether or not the individual purchases an indivisible "conspicuous" good. The good could be a pure positional good with no intrinsic value (an ugly abstract art piece), but also a fancy house or car. What matters is that its consumption is observable while the consumption of $C$ is not. The price of the composite good is normalized to 1 . For simplicity, we assume that the conspicuous good has elastic supply at price $\pi>0$. The consumer faces budget constraint $C+a \pi=x$, where $x$ is income. We assume that people care about consumption and the perception others have of their income, $x$. The total net utility (including esteem) from consumption of $a$ is thus $v(x ; \pi)+E(x \mid a=1)-E(x \mid a=0)$, where $v(x ; \pi) \equiv U(1, x-\pi)-U(0, x)$ is assumed to be increasing in $x$ (i.e. $a$ is a normal good) and sufficiently steep (so that Assumption 1 is satisfied). We also assume that the price $\pi$ is neither too low nor too high, so that an interior income cutoff $x^{*}$ exists. 
Consider now the introduction of a guaranteed minimum income $\underline{\underline{x}}$, so that all types below $\underline{\underline{x}}$ are guaranteed a post-policy income of $\underline{\underline{x}}$. This may be achieved by topping up their initial income through government transfers or by regulating/subsidising their wages, or through a mix of transfers and labour market interventions. We abstract from the details of funding sources, although it is worth noting that the introduction of a progressive income tax to fund the policy would generally strengthen the result we highlight below ${ }^{15}$

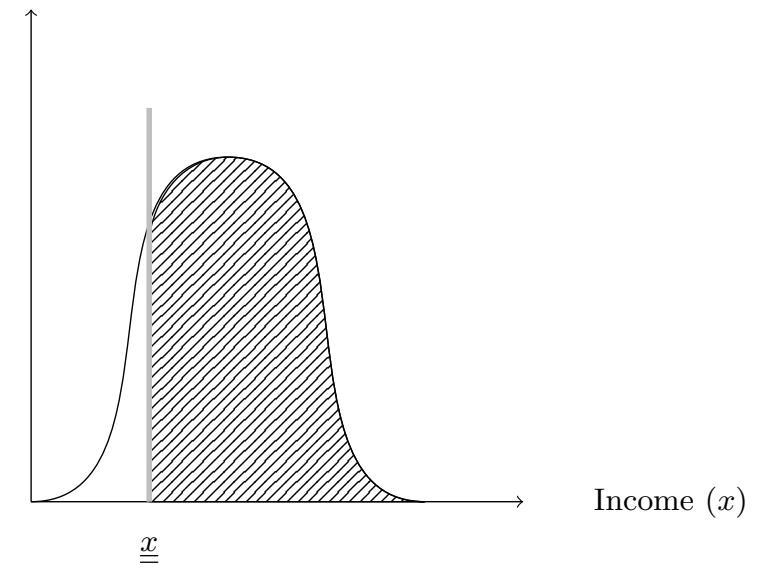

Figure 4: A guaranteed minimum income induces a left censoring of the pre-transfers income distribution.

As illustrated in Figure 4, the ex-post income distribution is a left censored version of the initial distribution. The mass in the left tail is moved to the right to create an atom at the minimum income $\underline{\underline{x}}$. How would this affect consumption choices? Proposition 2 implies that the propensity to consume the conspicuous good will be lower as a result of the policy. In other words, a guaranteed minimum income induces a reallocation of consumption away from the conspicuous good. Intuitively, by eliminating the left tail, the policy reduces the stigma associated with being pooled with the poor ${ }^{16}$

It is worth however emphasizing that - so long as the policy is implemented through direct transfers - the implicit assumption that agents are concerned about the perception of their post-transfers income is not innocuous. If agents cared instead only about their inferred

\footnotetext{
${ }^{15} \mathrm{~A}$ budget balanced redistributive policy is considered in Section 6

${ }^{16}$ Note that, while the individual propensity to consume the conspicuous good always drops, a reduction in aggregate demand requires that the minimum income $\underline{\underline{x}}$ is not so high as to fall above the (now increased) cutoff.
} 
initial income, the transfers would not change signaling incentives. In practice, this suggests that the means through which the policy is implemented may matter. For instance, the effect of the policy may be different if the guaranteed minimum income is mainly achieved through government handouts or through other tools like minimum wage regulation.

\subsection{Dispersion}

The result on truncations hints at the main theme of this section, namely that "fatter tails" increase the esteem gain from performing the action and thus the incentive to perform. We now illustrate this point by considering groups that can be ordered in terms of their dispersion. We thus assume that group $g$ and $g^{\prime}$ have identical means, but group $g$ 's distribution is second order stochastically dominated by group $g^{\prime}$.

Comparing $g$ and $g^{\prime}$ is not immediately obvious. However, as pointed out by Jewitt (2004), the function $\phi(x)$ can be expressed in terms of the Lorenz curve associated with the type distribution. Setting $p \equiv F(x)$,

$$
\phi(x)=E(X \mid X>x)-E(X \mid X<x)=\theta \frac{p-\mathcal{L}(p)}{p(1-p)} \equiv \Phi(p)
$$

where $\mathcal{L}(p) \equiv \frac{1}{\theta} \int_{0}^{p} F^{-1}(s) d s$ is the Lorenz curve. Consider then two groups, $g$ and $g^{\prime}$, with identical mean $\theta$. Assume that the Lorenz curve of group $g, \mathcal{L}_{g}$, lies below $\mathcal{L}_{g^{\prime}}$ for all $p \in(0,1)$. Clearly enough, $\Phi_{g}(p)>\Phi_{g^{\prime}}(p)$ for all $p \in(0,1)$. This in turn implies that $\phi_{g}(x)>\phi_{g^{\prime}}(x)$ for all $x \in\left(\underline{X}_{g}, \bar{X}_{g}\right){ }^{17}$ Since the two distributions have identical mean, saying that $\mathcal{L}_{g}$ lies below $\mathcal{L}_{g^{\prime}}$ is equivalent to saying that $f_{g}$ is second order stochastically dominated by $f_{g^{\prime}}$ (see e.g. Atkinson, 1970). This leads us to the following result,

Lemma 3. If $f_{g}$ and $f_{g^{\prime}}$ have identical mean and $f_{g}$ is second order stochastically dominated by $f_{g^{\prime}}$, then $\phi_{g}(x)>\phi_{g^{\prime}}(x)$ for all $x \in\left(\underline{X}_{g}, \bar{X}_{g}\right)$.

Proof. In text.

Lemma 3 establishes that esteem concerns are stronger when the group is more dispersed. Figure 5 provides a visual illustration of the result in Lemma 3 and its implications for incentive provision. These are summarized in the following result.

\footnotetext{
${ }^{17}$ Notice that the two groups need not have the same support. In principle, the support for the distribution of group $g^{\prime}$ could be a strict subset of that for group $g$.
} 

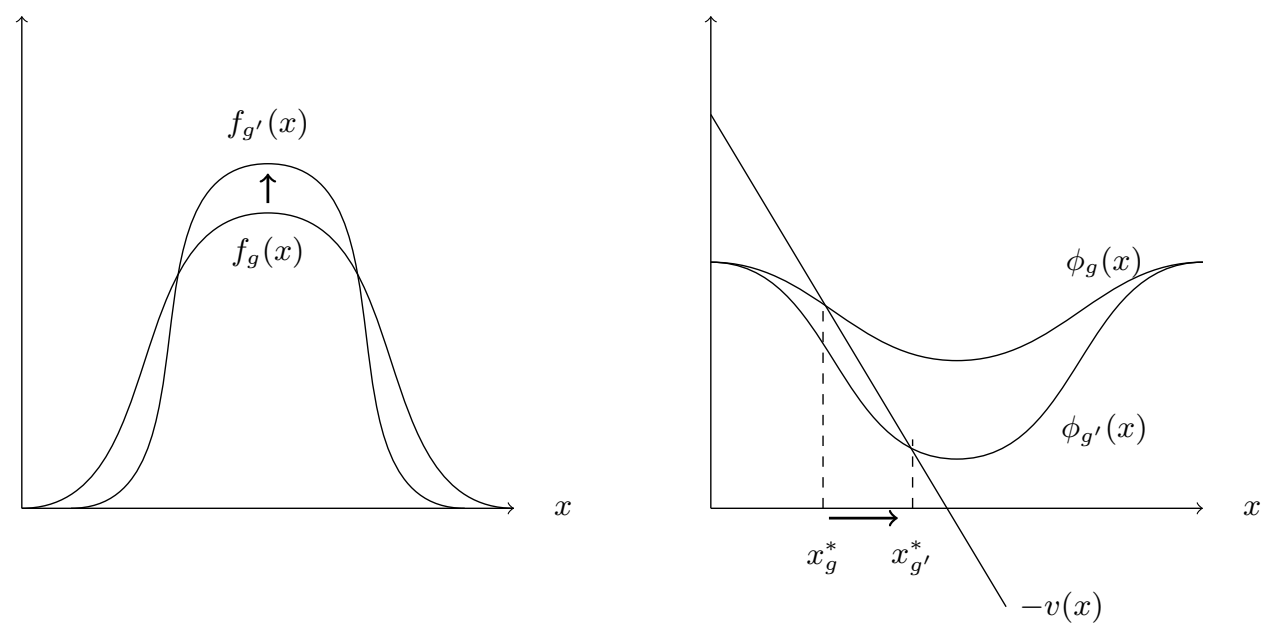

Figure 5: If $f_{g}$ is second order dominated by $f_{g^{\prime}}$, then $\phi_{g}$ lies above $\phi_{g^{\prime}}$.

Proposition 3. Consider two groups with densities $f_{g}$ and $f_{g^{\prime}}$ with identical mean. Then, if $f_{g}$ is second order dominated by $f_{g^{\prime}}$, the incentives to provide the signal are stronger in group $g$ than in group $g^{\prime}$, i.e. $x_{g^{\prime}}^{*}>x_{g}^{*}$.

Proof. Directly from Lemma 3 and the fact that the LHS of (3) is decreasing in $x$.

Proposition 3 thus establishes an unambiguous relationship between group dispersion and incentives. Intuitively, a more dispersed group increases the rewards from social signaling. In a more dispersed group, providing the signal means pooling with a fatter right tail of very high types and, at the same time, separating from a fatter left tail of extremely low types. It is straightforward to verify that any symmetric unimodal density second order dominates any symmetric U-shaped density with identical mean and the same (or larger) support. Applying Proposition 3 , this implies,

Corollary 2. Consider any two groups $g$ and $g^{\prime}$ with symmetric densities $f_{g}$ and $f_{g^{\prime}}$, both with mean $\theta$ and with $\left[\underline{X}_{g^{\prime}}, \bar{X}_{g^{\prime}}\right] \subseteq\left[\underline{X}_{g}, \bar{X}_{g}\right]$. Then, if $f_{g^{\prime}}$ is unimodal and $f_{g}$ is U-shaped, group g provides stronger incentives.

Example: Inequality and conspicuous consumption The example discussed in the previous section can now be used to shed light on the relationship between inequality and conspicuous consumption at a more general level 18 Given Proposition 3 , it is clear that the propensity to consume the "observable" consumption good $a$ is higher in groups with a more

\footnotetext{
${ }^{18}$ Bowles and Park (2005) suggest that the observed positive relationship between increased inequality and
} 
unequal distribution of wealth. Glazer and Konrad (1996) analyze a similar problem in a model where status is signalled by charity donations. The key difference with the present model is that they focus on a fully separating equilibrium (which is feasible in their case since donations are a continuous variable). In a fully separating equilibrium, the shape of the distribution does not affect the incentives of each type because the incentive compatibility constraints are independent of the types' frequencies. By converse, our result is entirely driven by (partial) pooling. Inequality increases the incentive to purchase the observable good by allowing one to pool with a fatter tail of super rich individuals (and to separate from a fatter tail of extremely poor individuals). This effect is therefore absent in their model 19 Moreover, while it seems natural to model charitable donations as a continuous variable, it is clear that many status symbols come in the form of "lumpy" indivisible goods (e.g. luxury watches, sports cars, branded suits) ${ }^{20}$

To conclude this section, we note what happens when the signal observed by the audience is imperfectly correlated with the action taken by the agent. This is analyzed in more detail in the supplementary appendix where we look at the example of a test whose outcome (pass, fail) is a noisy signal of the agent's effort. We establish there that, at least in the case where both types and noise are symmetrically distributed, a mean preserving spread has two mutually reinforcing effects on incentives ${ }^{21}$ One is the tail fattening effect discussed above. The second effect is due to the mean preserving spread increasing in the informative content of the noisy signal relative to the information conveyed by peer group membership.

longer working hours may be due to an increase in conspicuous consumption in order to "keep up with the Joneses" (see also Frank et al. 2005).

${ }^{19}$ In a model with continuous effort but discrete types, Lee (2007) shows that US college students exert more effort than their East Asian counterparts. The result is driven by the fact that, because of selection based on high school achievements, East Asian colleges are more homogeneous in terms of ability.

${ }^{20}$ Even in the case of donations, however, professional fundraisers typically find ways to make giving more or less visible according to whether the pledged amount fall above or below certain thresholds. For instance, membership of the exclusive Leader's Group of donors of the UK Tory party entitles the donor to participate in social events with senior party members and have his/her name listed on the party's website. A donor has access to these benefits only by making a donation of GBP 50,000 or more.

21 More precisely, we prove the result for the case where 1) the probability of passing when shirking is the same as the probability of failing when exerting effort; 2) Types are symmetrically distributed. When either 1) or 2) fail, further effects arise which may reduce the effect of a mean preserving spread in some situations. These effects are briefly discussed in the supplementary appendix. 
Intuitively, suppose that the group distribution is initially very concentrated around the mean. The type of any individual drawn from that distribution is thus likely to be in a small neighborhood of the mean. In that case, the posterior beliefs upon observing the result of a noisy test will be close to the mean, independently of whether the agent passes or fails. Following a mean preserving spread, however, the type distribution becomes more dispersed. As group membership becomes less informative, posterior beliefs become more sensitive to the result of the noisy test, thus providing stronger incentives.

\subsection{Skewness}

In this section, we analyze what happens when the peer distribution becomes more right or left skewed. The results here complement and, to some extent, generalize the observations already made for truncations. For convenience, we will rank location scale families of distributions according to how right skewed they are using the criterion proposed by Jewitt (2004). The basic idea is illustrated in Figure 6. Both $f_{g^{\prime}}$ and $f_{g^{\prime \prime}}$ belong to the same family and are more "right skewed" than $f_{g}$, in the sense that they have thinner left tails and fatter right tails. Note that distributions in the same location scale family as $f_{g^{\prime}}$ and $f_{g^{\prime \prime}}$ cross $f_{g}$ at most three times (as $f_{g^{\prime}}$ does) and, when three crossings occur, the first is from below.

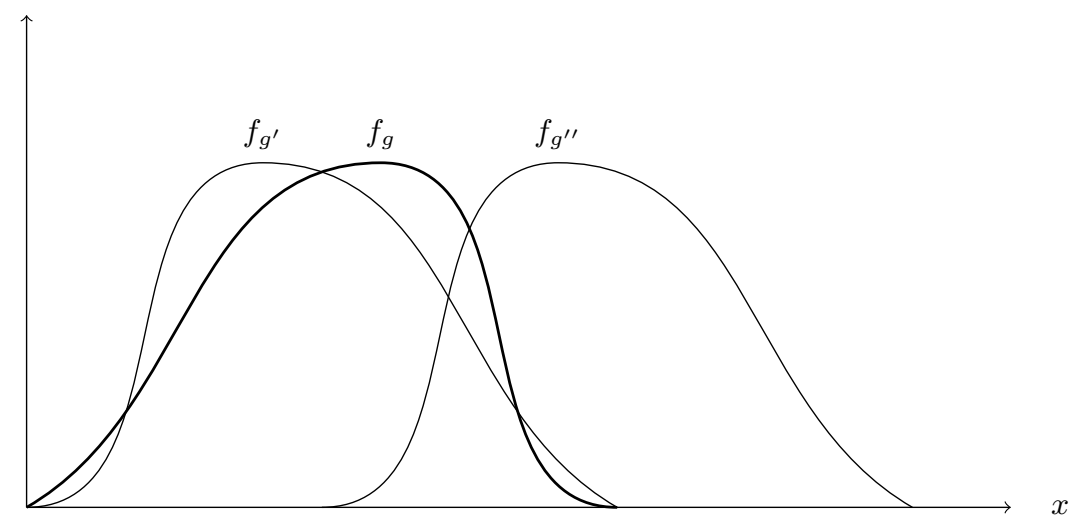

Figure 6: $f_{g^{\prime}}$ and $f_{g^{\prime \prime}}$ are more right skewed than $f_{g}$.

Definition 1. Let $f$ and $\tilde{f}$ be two densities and let $\mathbb{F}$ and $\tilde{\mathbb{F}}$ denote their respective location scale families. If $\mathbb{F}$ and $\tilde{\mathbb{F}}$ have the property that any pair of densities $(h, \tilde{h}) \in \mathbb{F} \times \tilde{\mathbb{F}}$ cross at most three times and that, when they do cross exactly three times, the first crossing has $\tilde{h} \in \tilde{\mathbb{F}}$ crossing $h \in \mathbb{F}$ from below, then $\tilde{f}$ is more right skewed than $f$. 
Jewitt (2004) shows that if a distribution is more right skewed than another according to the above criterion, then it also has a higher third standardized moment, although the reverse is not necessarily true.

In order to isolate the effect of skewness, it seems appropriate to compare distributions with identical mean (although not necessarily with identical support). In this case, how distributions compare in terms of their skewness (as defined above) has implications for the behavior of their Lorenz curves.

Lemma 4. (Jewitt, 2004) Suppose that $f_{g^{\prime}}$ and $f_{g}$ have the same mean, $\theta$, and that $f_{g^{\prime}}$ is more right skewed than $f_{g}$. Then, the Lorenz curves $\mathcal{L}_{g}(p) \equiv \frac{1}{\theta} \int_{0}^{p} F_{g}^{-1}(s) d s$ and $\mathcal{L}_{g^{\prime}}(p) \equiv$ $\frac{1}{\theta} \int_{0}^{p} F_{g^{\prime}}^{-1}(s) d s$ cross at most once for $p \in(0,1)$ and, if they do cross, $\mathcal{L}_{g}$ crosses $\mathcal{L}_{g^{\prime}}$ from above.

Proof. See appendix.

As already noted in the previous section, Lorenz dominance implies second order stochastic dominance for distributions with identical means. We are thus left with two possible outcomes. Either one of the two distributions is Lorenz-dominated - which means that it is also second order stochastically dominated - or the Lorenz curves of the two distributions must cross exactly once. In terms of the net esteem function $\Phi(p)$ given in (7), this implies,

Lemma 5. Suppose that $f_{g}$ and $f_{g^{\prime}}$ have the same mean and that $f_{g^{\prime}}$ is more right skewed than $f_{g}$. Then, either one distribution is second order dominated by the other, or there exists $\hat{p} \in(0,1)$ such that $\Phi_{g}(p)>\Phi_{g^{\prime}}(p)$ for all $p<\hat{p}$ and $\Phi_{g}(p)<\Phi_{g^{\prime}}(p)$ for all $p>\hat{p}$.

Proof. See appendix.

We already analyzed the effect of dispersion on incentives in the previous section. If one distribution is second order dominated by the other, then it provides uniformly stronger incentives. If we want to isolate the effect of skewness, we need to focus on the case where neither of the two distributions is second order dominated.

Proposition 4. Suppose that $f_{g}$ and $f_{g^{\prime}}$ have the same mean and cannot be ranked according to second order dominance, but $f_{g^{\prime}}$ is more right skewed than $f_{g}$. Then, there exists a critical mass of agents $\hat{p} \in(0,1)$ such that, if the share of abstainers is lower than $\hat{p}$ in both groups, 
group $g$ provides stronger incentives, i.e. $x_{g}^{*}<x_{g^{\prime}}^{*}$. If the share of abstainers is greater than $\hat{p}$ in both groups, then group $g^{\prime}$ provides stronger incentives, i.e. $x_{g}^{*}>x_{g^{\prime}}^{*}$.

Proof. See appendix.

Intuitively, a peer group with a more right skewed distribution provides stronger incentives when provision is rare, so that honor is the main concern. This is because, by providing the signal, one can pool with a fatter tail of very high types. By converse, when provision is widespread and the stigma from failing to provide the signal is the main worry, incentives are stronger under a more left-skewed distribution.

Example: Conspicuous Consumption and Inequality (continued) Rather than analyzing the effect of a mean preserving spread in the distribution of income, we can ask what happens when the income distribution becomes more or less right skewed. If the conspicuous good is very expensive (e.g. a yacht) so that a sufficiently small fraction of the population consumes it, a more right skewed distribution provides a stronger incentive to consume it. On the other hand, when it is relatively affordable, a more left-skewed distribution provides stronger incentives.

\section{DCS and aggregate signal provision}

The previous section looked at how incentives change following a change in the peer group distribution. In particular, we derived conditions under which the marginal type in group $g$ is smaller than in group $g^{\prime}$, so that all types who provide the signal under $g^{\prime}$ also provide the signal under $g$ and some types switch from abstention to provision. This is relevant for questions like: would moving a pupil to a more diverse school improve or weaken his academic record? How do existing members of a group (community, firm's employees, school) react to inflows or outflows of new and potentially different agents (e.g. because of immigration, mergers, changes in catchment area)? ?22

On the other hand, policy makers and empirical researchers may also be interested in more aggregate effects, like changes in the total share of abstainers vis-à-vis signal providers following changes in the distribution. For instance, in the context of conspicuous consumption, one may ask how the demand for the conspicuous good $1-F_{g}\left(x_{g}^{*}\right)$ is affected by the

\footnotetext{
${ }^{22}$ See Grout et al. (2015) for an analysis of these issues within the context of coordination problems.
} 
minimum income policy or by redistribution in general.

Under most manipulations of $f_{g}$, there will be two effects on aggregate provision. The first, which we call the incentive effect is the shift in the cutoff $x^{*}$ discussed so far. The second effect, which is essentially a composition effect, is purely mechanical. Keeping the cutoff constant, any manipulation of the distribution shifts the probability mass away from some types and toward other types - who may or may not provide the signal. For instance, a mean preserving spread of the income distribution would increase both the frequency of disadvantaged individuals and that of the well off, while reducing the frequency of agents with average income. Since signal provision is different across income levels, this will directly affect aggregate provision. The two effects may go in the same direction or may counteract each other. In this section we provide an account on how they interact. For reasons of space, some formal results are made available in the supplementary appendix.

\subsection{Uniform shifts}

The effect of uniform shifts on aggregate provision is analyzed in Bénabou and Tirole (2006, 2011). We refer the reader to their works. To summarize, right shifts of the distribution always increase aggregate provision. Bénabou and Tirole (2011) also note that the social multipliers may be larger than one (when there is strategic complementarity) or lower than one (strategic substitutability). In terms of our analysis, a multiplier lower than unity occurs whenever there is a small fish in a big pond effect, so that the incentive effect partially offsets the composition effect. Symmetrically, a multiplier larger than one occurs when the incentive effect reinforces the composition effect.

\subsection{Truncations, censoring and group splits}

For the purposes of analyzing aggregate provision, it is necessary to distinguish between two types of manipulations,

1. A "proper" truncation or a "proper" censoring in the sense that the truncated/censored group has the same size as the initial group 23

\footnotetext{
${ }^{23}$ If $f_{g^{\prime}}$ is obtained through a left truncation/censoring of $f_{g}$, then the total mass of agents is $\int_{k}^{\bar{X}_{g}} f_{g^{\prime}}(x) d x=$ $\int_{\underline{X}_{g}}^{\bar{X}_{g}} f_{g}(x) d x=1$. The same applies to right truncations/censoring, but clearly does not hold when the initial group is split into smaller subgroups.
} 
2. A mere group split, which divides the initial group into two smaller subgroups of size $F_{g}(k)$ and $1-F_{g}(k)$, respectively

The first case can be thought of as the case of a school introducing screening at entry level, while keeping the number of pupils constant. The minimum income application similarly involves a proper censoring. The second case applies if a class of pupils is split into two smaller subgroups based on ability. We look at both these cases in the supplementary appendix. For group splits, the composition effect is largely immaterial. It is thus easy to apply Proposition 2 to establish that, so long as at least one of the two subgroups has an interior cutoff, overall provision across both subgroups must fall (see Proposition 12). In the first case, the composition effect plays a more complex role. For example, the left censoring described in the minimum income application takes the mass of poor agents in the left tail and reallocates it at the minimum income, keeping group size constant. Focusing again on the non-degenerate case where the ex-post cutoff is interior to the truncation/censoring, our results confirm the intuition that both left and right censoring always lead to lower aggregate provision (see Proposition 13). The same applies to right truncations, but not necessarily to left truncations. For left truncations, the incentive and composition effects go in opposite directions. Intuitively, one can think of a left truncation as if the mass in the left tail below

$k$ were "spread" over all types in the $\left[k, \bar{X}_{g}\right]$ support, including types above the cutoff $x_{g^{\prime}}^{*}$ who provide the signal. Hence, while peer pressure is weaker, the changed composition may favor higher provision.

In terms of implications, this suggests that, keeping the overall number of students constant, if the top students are moved out of a school, aggregate school performance always decreases (both because of weaker incentives and because of the higher frequency of relatively weak students). However, if the worst students are removed, aggregate school performance may go up or down depending on the strength of the incentive effect.

\subsection{Dispersion}

As shown above, a mean preserving spread unambiguously increases esteem incentives. However, a mean preserving spread also tends to shift the mass away from the median and towards the tails of the distribution. In the conspicuous consumption application, this implies more super rich but also more super poor (who may struggle to afford the conspicuous good). As 
a result, the overall effect on aggregate provision is not a priori clear.

It is known that second order dominance imposes little structure on the relationship between two densities, so that, outside the convenient cases where the mapping from types into actions is a concave or convex function, not much can be said about aggregate effects. Similar to most of the literature, we thus build intuition by imposing a more precise structure on how the two distributions relate to each other 24 We start with the case where the two densities cross only twice (so that the CDFs cross only once), as depicted in Figure 5 (and as in the standard textbook description of a mean preserving spread) ${ }^{25}$ The next result establishes that the composition effect reinforces the incentive effect when signal provision is rare, so that the aggregate effect of greater dispersion is unambiguous. In contrast, the two effects go in opposite directions if signal provision is widespread (since more dispersion makes the left tail heavier).

Proposition 5. Suppose that $f_{g}$ and $f_{g^{\prime}}$ have the same mean and $f_{g}$ is second order dominated by $f_{g^{\prime}}$, with the two densities crossing exactly twice. Then, there exists a unique value for the proportion of abstainers $\hat{p} \in(0,1)$ satisfying $F_{g}^{-1}(\hat{p})=F_{g^{\prime}}^{-1}(\hat{p})$. If the actual proportion of abstainers in group $g$ is larger than the critical value $\hat{p}$, then aggregate provision will be larger in group $g$ than in $g^{\prime}$. If the actual proportion of abstainers in group $g$ is smaller than $\hat{p}$, then aggregate provision will be larger in group $g^{\prime}$ when $v(x)$ is sufficiently steep for types $x<F_{g}^{-1}(\hat{p})$.

Proof. See appendix.

Remark 1. If $f_{g}$ and $f_{g^{\prime}}$ are both symmetric, then $\hat{p}=1 / 2$, i.e. it is sufficient that provision is minoritarian to ensure that it is larger in group $g$ than in $g^{\prime}$.

Example: dispersion and demand for the conspicuous good Consider how the demand for the conspicuous good $1-F_{g}\left(x_{g}^{*}\right)$ is affected by a mean preserving spread of the income distribution. Intuitively, if the conspicuous good is very expensive, so that only a minority can afford it, then more inequality implies both stronger incentives and a heavier

\footnotetext{
${ }^{24}$ For instance, Hopkins and Kornienko (2004) propose a unimodal likelihood ratio ordering to strengthen second order dominance. Levy and Razin (2015) use instead the concept of monotone mean preserving contraction

${ }^{25}$ For densities with the same support, the requirement that they only cross twice is implied by the Unimodal Likelihood Ratio ordering proposed by Hopkins and Kornienko (2004).
} 
tail of super rich with the means to provide the signal. However, if conspicuous consumption is widespread, so that stigma is the agents' main concern, then a more unequal society may induce higher or lower demand. The result in this case depends on how steep is the $v$ function for low income individuals. Intuitively, if $v$ is approximately vertical, the cutoff does not move much and the incentive effect is negligible. At the same time, the fattening of the left tail reduces provision. The slope of $v$ can be shown to depend on two factors: 1) the affordability of the conspicuous good for individuals in the lower tail and 2) the strength of the complementarity in consumption between the conspicuous good and other goods. Conspicuous goods that absorb a large fraction of income for the lower tail and that are more attractive when consumed jointly with the composite good will exhibit a steeper $v$ function $\sqrt{26}$ We can thus broadly identify three scenarios, depending on the exact nature of the conspicuous good.

1. The conspicuous good is an elite good (e.g. private yachts). In this case, the demand for the conspicuous good will increase as inequality increases.

2. The conspicuous good complements other consumption and, while widespread, involves relatively large expenditures (e.g. private vehicles). In this case, the demand for the good will generally be inversely related to inequality.

3. The conspicuous good is affordable and does not complement other consumption (e.g. nail salon services). In this case, demand will typically increase with inequality.

In the supplementary appendix, we extend the result in Proposition 5 by ditching the two density crossings requirement and assuming more generally that the the CDFs cross an odd number of times. Further insights on the relationship between dispersion and aggregate signal provision can be obtained if we follow Glazer and Konrad (1996) and take a parametric

\footnotetext{
${ }^{26}$ The derivative of $v(x)$ can be decomposed into$$
\frac{d v}{d x}=\left[\frac{\partial U(1, x-\pi)}{\partial x}-\frac{\partial U(1, x)}{\partial x}\right]+\left[\frac{\partial U(1, x)}{\partial x}-\frac{\partial U(0, x)}{\partial x}\right]
$$

The first term in brackets is large when the marginal utility from the composite goods is sharply decreasing (e.g. consuming the conspicuous good implies below subsistence consumption of other goods) and/or the amount of composite good that needs to be given up is large. The second term is large when the two goods are strong complements.
} 
approach. The following result analyzes how aggregate provision reacts to an increase in standard deviation keeping the mean constant.

Proposition 6. Let $x=\theta+\sigma z, \sigma \in \mathbb{R}^{+}$, where $E(z)=0, \operatorname{Var}(z)=1$ (so that $E(x)=\theta$ and $\left.\operatorname{Var}(x)=\sigma^{2}\right)$.

1. Suppose that the signal is costly for the mean type $(v(\theta)<0)$. Then, there exists $\epsilon>0$ such that, if $v^{\prime \prime}(x)>-\epsilon$ for all $x$ (i.e. $v$ is not too concave), aggregate provision is increasing in $\sigma$.

2. Suppose that the mean type intrinsically benefits from providing the signal $(v(\theta)>0)$. Then, there exists $\epsilon>0$ such that, if $v^{\prime \prime}(x)<\epsilon$ for all $x$ (i.e. $v$ is not too convex), aggregate provision is decreasing in $\sigma$.

Proof. See appendix.

Suppose first that $v$ is linear, as for instance in Bénabou and Tirole (2011). In this case, Proposition 6 provides necessary and sufficient conditions for an increase of the standard deviation to result in an increase in aggregate provision. This happens if and only if the signal is costly for the mean type. To see the intuition, it is useful to think about what happens when the standard deviation decreases. This has two effects. First, the mass of agents is "pushed" toward the mean. Second, esteem concerns are weaker. If the mean type would provide the signal even in the absence of image concerns, such a contraction of the distribution can only increase aggregate provision. However, in the more standard case where the signal is costly for the mean type, so that he would not provide it absent image concerns, then the contraction results in a reduction of aggregate provision. More generally, if the signal is costly for the mean type, aggregate provision increases in the standard deviation if $v^{\prime}(x)$ is not too decreasing. This is needed to ensure that $v(x)$ is never too steep for types in the left tail, so that, when the share of abstainers is low, the cutoff is sufficiently sensitive to changes in $\sigma$. In other words, the incentive effect dominates. The opposite happens when the mean type intrinsically benefits from providing the signal and $v^{\prime}(x)$ is non-increasing. We will come back to this result in Section 6 when we will discuss the effects of redistribution in more detail. 


\subsection{Skewness}

The interaction between the incentive effect and the composition effect as the distribution becomes more right skewed is quite complex. A detailed analysis is provided in the supplementary appendix. We show there that the two effects go in the same direction when the share of abstainers is sufficiently large (toward increased aggregate provision) and for intermediate values of the share of abstainers (toward reduced provision). In general, as seen in Proposition 4, the incentive effect favors higher provision if the proportion of abstainers is above a certain level. In contrast, the composition effect favors higher provision if the proportion of abstainers is either very high or very low, while it induces lower provision for intermediate levels of abstention.

\section{Welfare and redistribution under purely wasteful signals}

In this section, we focus on the conspicuous consumption application to ask several questions about welfare: is redistribution beneficial? When does a majority in favor of redistribution exist? How do taxes and transfers affect the incentive to provide the signal? In order to cast our results in stark and clear terms, we focus on the case where the signal is wasteful. In particular, we assume $U(1, x)=u(x)$ and $U(0, x)=u(x-\pi)$ with $u: \mathbb{R}^{+} \rightarrow \mathbb{R}^{+}$strictly increasing and $\pi>0$ denoting the price of the conspicuous good ${ }^{27}$ The cost of the signal is thus $-v(x)=u(x)-u(x-\pi)$ which is positive for all $x \in[\underline{X}, \bar{X}]$. As a first step, we follow Levy and Razin (2015) and analyze a full redistribution (FR) scheme under which each individual in the peer group faces a $100 \%$ tax rate and is provided a transfer equal to the group's mean income $\theta$. Clearly enough, in the presence of FR there is no incentive to provide the costly signal. We note the following facts,

1. FR is socially optimal whenever the marginal utility of income is non-increasing, i.e. $u$ is (weakly) concave.

2. Even if FR is optimal, a majority supporting FR need not exist.

3. When the mean, $\theta$, is weakly larger than the median, $\mu$, sufficient conditions for a

\footnotetext{
${ }^{27}$ We implicitly assume that agents with $x<\pi$ have no choice but to abstain.
} 
majority in favor of $\mathrm{FR}$ are that either $x^{*} \geq \mu$ (i.e. the median type abstains) or that

$$
\frac{\phi(\mu)}{2}<u(\theta)-u(\mu-\pi)
$$

All these statements are formally established in the supplementary appendix, where we also provide sufficient conditions for the less empirically relevant case where the median is above the mean. The logic for the first statement is standard. Since the signal is wasteful, it is sufficient that the utility of income is not convex to ensure that FR is optimal. As for statement 2 , we work out in the supplementary appendix a counterexample with log utility and uniformly distributed income where for parameter values the majority opposes FR. This seems to happen when the price of the conspicuous good is low - so that signal provision is widespread - and agents are more concerned with esteem than with material consumption. Intuitively, under these circumstances, the median income agent and those slightly below are willing to bear the cost of the signal (and possibly forgo higher post transfer income) in order to separate themselves from those in the low tail. Statement 3 generalizes these insights. To gather intuition on condition (9), consider first the case of symmetric distributions, so that $\theta=\mu$. In this case, (9) reduces to

$$
\frac{\phi(\mu)}{2}<-v(\mu)
$$

The RHS is the cost of the signal to the mean/median type in terms of forgone utility from consumption. The LHS is the extra esteem obtained from pooling with the right tail relative to being perceived as mean/median, when the median is the marginal type ${ }^{28}$ Back to (9), relative to the case of a symmetric distribution, a more skewed distribution (in the sense of $\theta$ much larger than $\mu$ ) makes FR easier to sustain. A less skewed distribution makes it harder. These effects are also standard (see e.g. Meltzer and Richard, 1981). Finally, FR can always be sustained if those who provide the signal in equilibrium are a minority and the mean is above the median. Intuitively, in this case all individuals with income below the median abstain in equilibrium. FR makes them better off both in terms of post transfer income - since their income is less than the mean income - and esteem - since they do not face stigma.

Having analyzed FR, we now turn to a different question. We have already seen in the previous sections that a compression of the distribution does not necessarily lead to fewer

\footnotetext{
${ }^{28}$ Note that, for symmetric densities, $E[x \mid x>\theta]-\theta=\phi(\theta) / 2$.
} 
agents providing the costly signal. Consider then a scheme where each individual faces a tax rate $t \in(0,1)$ and is provided by the government a handout equal to $t \theta$, so that the scheme balances the budget. We can build on the result in Proposition 6 to ask how such a scheme would affect signal provision.

Proposition 7. Aggregate provision of the wasteful signal is not generally decreasing in the intensity of redistribution, as measured by $t$. However, there always exists $\hat{t} \in(0,1)$ such that aggregate provision is decreasing in $t$ for all $t \geq \hat{t}$. Monotonicity for all $t \in(0,1)$ obtains whenever $v(x)$ is weakly convex and/or the price of the conspicuous good $\pi$ is larger than $\theta$. Proof. See supplementary appendix.

The first statement says that redistribution does not necessarily reduce the aggregate distortion due to signaling. In the supplementary appendix, we provide an example based on $\log$ utility and a uniform income distribution. Intuitively, as laid out in the previous sections, redistribution has both an incentive effect and a composition effect. Increasing taxes and transfers reduces the incentive for status seeking. On the other hand, it may make the costly signal affordable to the less well off. The second effect may in some cases outweigh the first. Essentially, this result echoes the concerns of some moralists since Victorian times that cash handed out to the masses may end up being wasted on "frivolous" consumption. In contrast, the second statement says that, although redistribution may actually increase the share of those purchasing the conspicuous good, this can only happen if there is too little redistribution in the first place. For groups that are already sufficiently egalitarian the effect is unambiguous and goes in the direction of reducing aggregate conspicuous consumption. The last statement says that the effect is similarly unambiguous if either $v(x)$ is convex so that $-v(x)$ is relatively flat for low income agents - or the conspicuous good is an elite good which is affordable only by the rich. Intuitively, the first condition ensures that the composition effect is never too strong. The second condition instead makes sure that the composition effect reinforces the incentive effect.

More generally, as shown in the proof of Proposition 7, if society is sufficiently egalitarian, the marginal type will be in the right tail. When this happens, the median type and everyone below support FR unless the income distribution is extremely left skewed. This implies that, while the majority may not always support FR, this is generally the case if inequality is 
already low. This echoes the results obtained in Levy and Razin's (2015) sorting model, and points to a "self sustaining" process, whereby redistribution breeds appetite for further reduction of inequality. An important difference between the two frameworks is that, in our model, the esteem reward from (partial) separation is the same for all types. In contrast, in Levy and Razin's model, there are complementarities between an individual's income and that of those he interacts with, so that higher types benefit more from separation. This implies that the welfare gains from sorting/separating tend to be larger than in the case of the honor-stigma model. For this reason, much of their focus is on cases where FR is supported even when inefficient. In our case, the opposite problem arises ${ }^{29}$

\section{Peer pressure under endogenous group formation}

So far, we have considered the case where individuals were exogenously assigned to a peer group. In practice, individuals may have some freedom in choosing their peers within a given set of potential peers. For instance, pupils attending a local school will typically sort themselves into cliques of friends, social clubs, etc. The main result of this section is that this endogenous sorting does not affect signaling incentives so long as 1) types are private information, and 2) status seeking is the only reason for joining a group. In particular, so long as groups can only restrict membership based on observable behavior, then all equilibrium allocations of individuals into groups (including the one where all individuals join the grand coalition) provide the same incentives. It thus makes sense to analyze incentives taking the (exogenously given) grand coalition as the relevant peer group.

Consider a continuum of individuals, $G$, with density $f_{G}$. In addition to choosing an action, each individual must join one of $N \geq 1$ groups indexed by $g=1, \ldots, N$. We allow for group membership to be restricted based on observable behavior, $a$, but we assume that there exists at least one group that can be joined by anyone independently of his action. A group structure is a rule assigning types to groups. Let $S:\left[\underline{X}_{G}, \bar{X}_{G}\right] \rightarrow \Delta$, where $\Delta$ is the set of probability distributions over $\{1, \ldots, N\}$, denote a group structure. We say that $S$ is an equilibrium group structure if each individual weakly prefers his group to any other

\footnotetext{
${ }^{29}$ For instance, in our model, the majority might prefer the status quo over FR even when FR is socially optimal and, as it happens in reality, median income is below mean income. In contrast, this never occurs in the case of sorting.
} 
group. Finally, let $S_{G}$ denote a structure where all individuals join a single group (the grand coalition). The next result establishes that any equilibrium group structure is equivalent, in terms of action provision, to a structure where all individuals join the grand coalition.

Proposition 8. For any PBE under a given equilibrium group structure $S$, there exists an outcome equivalent $P B E$ under $S_{G}$.

Proof. See Appendix.

Equilibrium requires that an individual choosing either action must be indifferent between joining any group where that action is played by a positive measure of agents (otherwise some agent would profit from joining a different group). However, for this to occur, the equilibrium posterior must be - conditional on performing or not the action - independent of group membership. This ensures that endogenous group membership provides no further information beyond the information already conveyed through the action choice. The result should be read as saying that, for group structure to have an effect on incentives, either individuals must have further reasons beyond status for joining a particular group or groups must screen members based on information beyond publicly observable behavior. Notice however that, in both these cases, group structure is at least partially determined by exogenous considerations.

\section{Further considerations on peer pressure and signaling}

The analysis of peer pressure through the lens of esteem concerns delivers some sharp predictions. Heterogeneous peer groups always provide stronger motivation than homogeneous groups. Similarly, agents whose peer distribution is truncated (e.g. because of segregation by ability) always experience weaker motivation. We also found that better peers boost or reduce motivation depending on the share of agents providing the signal and on whether the peer distribution is unimodal or not. In a similar fashion, conditional predictions can be obtained by manipulating the skewness of the peer distribution.

Clearly enough, our theory relies on the implicit assumption that the audience has accurate information about the true distribution of types in the group. An alternative interpretation of the comparative statics presented above is that, rather than the actual distribution 
changing, agents who initially lack precise information about a group are provided with a better estimate of the group distribution. For instance, if an agent overestimates the degree of inequality, a government report releasing information about the actual income distribution has analogous effects on his incentives as an actual contraction of the income distribution ${ }^{30}$ This interpretation closely follows Bénabou and Tirole (2011), who focus on the role of policies involving the strategic release of information to agents in addition to or in place of more traditional policies. Note however that, under this interpretation, there would be no composition effect (since the actual distribution does not change) and changes in aggregate provision would only reflect changes in incentives.

From a more theoretical viewpoint, our analysis helps to shed light on some, perhaps less well understood, features of the honor-stigma model.

Esteem and positionality We have noted that the net gain in esteem from performing the status enhancing action, $\Phi(p)$ in (7), depends on the share of agents who abstain in the reference group, $p$. In other words, the net gain in esteem depends on the "relative position" within the group assigned to those who perform the action. Consider then what happens if we add a positive constant to the type $x$ of each agent in the group, while keeping $p$ fixed. So long as $p$ does not change, this would have no effect on the net esteem gain from performing. Intuitively, the extra honor that can now be reaped through providing the signal is exactly offset by the lower stigma associated with abstaining. Overall, this suggests that assuming a concern for esteem is akin to introducing an element of positionality in the preferences. Two issues then immediately arise: First, is this true only of uniform shifts or does it also apply to other manipulations of the peer distribution? Second, is it possible to empirically discriminate between the honor-stigma model and a model where agents directly care about their perceived rank/position (rather than their perceived type)?

Perceived rank or perceived type? Consider the first question. The above remark on positionality holds because a uniform shift of the distribution affects $\Phi(p)$ only through $p$. The same is however not true if a shock affects other features of the distribution, like dispersion or skewness, which determine the shape of the Lorenz curve. In these cases,

\footnotetext{
${ }^{30}$ Note that, in this case, agents with rational expectations would also learn from their own type, so that beliefs would be heterogeneous across types. This implies that any strengthening or weakening of incentives may differ from type to type.
} 
signaling incentives will change even as we keep the share of abstainers constant. Intuitively, suppose that a period of economic growth increases the average income of the top $10 \%$ while the average income of the rest stagnates. In a perceived type model, like the present one, the incentive to signal that one is in the top $10 \%$ becomes stronger. If instead agents only care about perceived rank, being in the top $10 \%$ has the same value independently of how unequal society is. In other words, in perceived type models, identical individuals with the same perceived rank but in groups with distributions of different shape will typically experience different degrees of peer pressure.

We can go further and ask how the model predictions would change if image concerns were based on perceived rank rather than on type ${ }^{31}$ To fix ideas, consider a model where net esteem is given by $E[F(X) \mid X>x]-E(F(X) \mid X<x]$, with the cumulative $F(x)$ measuring a type $x$ agent's (unobservable) rank. It is easy to verify that, in this case, net esteem is always constant with respect to the shape of $F{ }^{32}$ As a result, changes in the shape of the distribution would not affect esteem incentives. This suggests that, in a rank based model, a mean preserving spread would only have a composition effect. When the data allow to control for the agent's type (for instance by using a "within subjects" approach), the presence of an incentive effect would thus be sufficient to discriminate between the two models. If, on the other hand, only aggregate provision were observable, one would need to look for cases where the composition effect and the incentive effect go in opposite directions (e.g. when the marginal type is in the left tail). This could in principle provide an indirect way to qualitatively assess the merits of the two approaches.

\section{A Appendix}

Proof of Lemma 1 Points a) and b) are established in Jewitt (2004). We now prove c) using an argument mirroring the one provided by Harbaugh and Rasmussen (2014). Let $m_{k}^{+}(x)$ and $m_{k}^{-}(x)$ denote the $k$-th derivatives of $m^{+}(x)=E(X \mid X>x)$ and $m^{-}(x)=E(X \mid X<x)$, respectively. [Since the peer group is fixed, we omit the $g$ argument.] We first need to

\footnotetext{
${ }^{31}$ We thank an anonymous reviewer for suggesting what follows.

${ }^{32}$ For any continuous $\mathrm{CDF}, F$, the random variable $y \equiv F(x)$ is always uniformly distributed in $[0,1]$ independently of the shape of $F$.
} 
establish that, for $f$ U-shaped, $\lim _{x \rightarrow \underline{X}} m_{1}^{-}(x)=\lim _{x \rightarrow \bar{X}}=1 / 2$. Note that,

$$
m_{1}^{+}(x)=\frac{f(x)}{1-F(x)}\left[m^{+}(x)-x\right], m_{1}^{-}(x)=\frac{f(x)}{F(x)}\left[x-m^{-}(x)\right]
$$

Consider the limit for $m_{1}^{-}$first. Using l'Hôpital,

$$
\begin{aligned}
\lim _{x \rightarrow \underline{X}} m_{1}^{-}(x) & =\lim _{x \rightarrow \underline{X}} \frac{f(x) \int_{\underline{X}}^{x} F(s) d s}{F(x)^{2}} \\
& =\lim _{x \rightarrow \underline{X}} \frac{f^{\prime}(x) \int_{\underline{X}}^{x} F(s) d s+f(x) F(x)}{2 F(x) f(x)} \\
& =\frac{1}{2}+\lim _{x \rightarrow \underline{X}} \frac{f^{\prime \prime}(x) \int_{\underline{X}}^{x} F(s) d s+f^{\prime}(x) F(x)}{2 F(x) f^{\prime}(x)+2 f(x)^{2}}=\frac{1}{2}
\end{aligned}
$$

where the last equality comes from the fact that, since $f$ is U-shaped, $f(\underline{X})>0$. A symmetric argument establishes that the right limit of $m_{1}^{+}$is also $1 / 2$.

In order to show that $\phi$ is quasi-concave, we need to show that $m_{2}^{+}(x)-m_{2}^{-}(x) \leq 0$ for all $x$ such that $m_{1}^{+}(x)-m_{1}^{-}(x)=0$. Note that

$$
\begin{array}{r}
m_{2}^{+}(x)=\frac{f^{\prime}(x) m_{1}^{+}(x)}{f(x)}+\frac{f(x)}{1-F(x)}\left[2 m_{1}^{+}(x)-1\right], \\
m_{2}^{-}(x)=\frac{f^{\prime}(x) m_{1}^{-}(x)}{f(x)}+\frac{f(x)}{F(x)}\left[1-2 m_{1}^{-}(x)\right]
\end{array}
$$

Consider first any $x$ such that $m_{1}^{+}(x)=m_{1}^{-}(x) \leq 1 / 2$. Then $m_{2}^{+}(x)-m_{2}^{-}(x) \leq 0$ as required. Consider now any $x$ such that $m_{1}^{+}(x)=m_{1}^{-}(x)>1 / 2$. If $x$ is smaller than the anti-mode, then $f$ must be decreasing. Given $m_{1}^{-}(x)>1 / 2$ and $f^{\prime}(x) \leq 0$, then $m_{2}^{-}(x)<0$ from (13). However, since $\lim _{x \rightarrow \underline{X}} m_{1}^{-}(x)=1 / 2, m_{2}^{-}(x)<0$ implies $m_{1}^{-}(x) \leq 1 / 2$, so that we obtain a contradiction. Symmetrically, consider any $x$ to the right of the anti-mode, so that $f$ is increasing. Then, $m_{1}^{+}(x)>1 / 2$ and $f^{\prime}(x) \geq 0$ imply $m_{2}^{+}(x)>0$. However, we know that $\lim _{x \rightarrow \bar{X}} m_{1}^{+}(x)=1 / 2$, so that we obtain a contradiction. This implies that there is no $x$ such that $m_{1}^{+}(x)=m_{1}^{-}(x)>1 / 2$.

Proof of Lemma 2 We provide the proof for the unimodal case, the U-shaped case being perfectly symmetric. From Lemma 1, $\phi(x)$ cannot have a (strict) interior maximum. [Again, since the peer group is fixed, we omit the $g$ subscript.] It follows that there exists some $x_{\text {min }}$ such that $\phi(x)$ is weakly decreasing to the left of $x_{\min }$ and weakly increasing to the right. [If $\phi(x)$ is monotonic in the support, take $x_{\min }=\arg \min _{x \in\{\underline{X}, \bar{X}\}} \phi(x)$.] We must rule out ranges of values for $x$ where $\phi(x)$ is constant. Notice now that

$$
\frac{d}{d x} \phi(x)=\frac{f(x)}{1-F(x)}\left[m^{+}(x)-x\right]-\frac{f(x)}{F(x)}\left[x-m^{-}(x)\right]
$$


Hence, $\phi(x)$ is increasing (decreasing) whenever

$$
\phi(x)>(<) \frac{x-m^{-}(x)}{F(x)}
$$

Suppose now that there exists an interval $\left(x^{\prime}, x^{\prime \prime}\right)$ such that $\phi(x)$ is constant for all $x \in$ $\left(x^{\prime}, x^{\prime \prime}\right)$. This implies that (15) holds with equality for all $x \in\left(x^{\prime}, x^{\prime \prime}\right)$. However, since $\phi(x)$ is constant in this interval, the RHS of 15 must also be constant for all $x \in\left(x^{\prime}, x^{\prime \prime}\right)$. Otherwise, 15) cannot hold with equality for all $x \in\left(x^{\prime}, x^{\prime \prime}\right)$. Notice that

$$
\frac{d}{d x}\left(\frac{x-m^{-}(x)}{F(x)}\right)=0 \Rightarrow \frac{x-m^{-}(x)}{F(x)}=\frac{1}{2 f(x)}
$$

for all $x \in\left(x^{\prime}, x^{\prime \prime}\right)$. But this in turn implies that, for all $x \in\left(x^{\prime}, x^{\prime \prime}\right)$,

$$
\phi(x)=\frac{1}{2 f(x)}
$$

However, $f(x)$ is always strictly increasing or decreasing (except exactly at the mode), which contradicts the claim that $\phi(x)$ is constant within any interval of positive measure. Hence, $\phi(x)$ must be strictly decreasing to the left of $x_{\min }$ and strictly increasing to the right. [Notice that this also rules out the uniform case where $\phi(x)$ is always constant.] We now show that the minimum must coincide with the mode for $f$ symmetric. Let $Z=X-\theta$. Notice that, from symmetry,

$$
\begin{aligned}
m^{+}(x)=\theta+E(Z \mid Z>x-\theta) & =\theta+E(-Z \mid Z<\theta-x)= \\
2 \theta-E(X \mid X<2 \theta-x) & =2 \theta-m^{-}(2 \theta-x)
\end{aligned}
$$

As a result, $\phi(x)=\phi(2 \theta-x)$. However, this implies that if $x \neq \theta$ minimizes $\phi$, then also $2 \theta-x$ minimizes $\phi$, which contradicts the previous result of a unique minimum. Hence, $\phi(x)$ is only minimized for $x=\theta$. Finally, a symmetric argument can be made for the U-shaped case.

Proof of Proposition 1 Since $f_{g}$ and $f_{g^{\prime}}$ differ only in the mean and have exactly one change in monotonicity, $\phi_{g}$ and $\phi_{g^{\prime}}$ can cross at most once. If the densities are symmetric and unimodal, then $\phi_{g}$ and $\phi_{g^{\prime}}$ have their minima at $\theta_{g}$ and $\theta_{g^{\prime}}$, respectively, and must cross once. The crossing point must thus be in the interval $\left(\theta_{g}, \theta_{g^{\prime}}\right)$. The same argument (using maxima instead of minima) applies to symmetric U-shaped densities. Consider then any shift in densities such that $\phi_{g}$ and $\phi_{g^{\prime}}$ cross and let $\hat{x}$ be the unique crossing point. In the unimodal 
case we will thus have $\phi_{g}(x)>\phi_{g^{\prime}}(x)$ for $x>\hat{x}$ and $\phi_{g}(x)<\phi_{g^{\prime}}(x)$ for $x<\hat{x}$. The opposite applies to the U-shaped case. Evidently, from Assumption 1, the cutoffs of both groups must lie on the same side of $\hat{x}$, so that (i) must hold. Results (ii) and (iii) then follow from the fact that the RHS of (3), $-v(x)$, is strictly decreasing. Consider now the case where $\phi_{g}$ and $\phi_{g^{\prime}}$ never cross. Since $\bar{X}_{g}>\underline{X}_{g^{\prime}}$, this can only happen if $\phi_{g}\left(\underline{X}_{g}\right)=\phi_{g^{\prime}}\left(\underline{X}_{g^{\prime}}\right) \neq \phi_{g}\left(\bar{X}_{g}\right)=$ $\phi_{g^{\prime}}\left(\bar{X}_{g^{\prime}}\right)$. Suppose first that $\phi_{g}\left(\underline{X}_{g}\right)=\phi_{g^{\prime}}\left(\underline{X}_{g^{\prime}}\right)>\phi_{g}\left(\bar{X}_{g}\right)=\phi_{g^{\prime}}\left(\bar{X}_{g^{\prime}}\right)$. Then, given no crossings, $\phi_{g}$ must necessarily lie below $\phi_{g^{\prime}}$, i.e. $\phi_{g}(x)<\phi_{g^{\prime}}(x)$ for all $x \in\left[\underline{X}_{g^{\prime}}, \bar{X}_{g}\right]$. Since the RHS of (3) is strictly decreasing, then $x_{g^{\prime}}^{*}<x_{g}^{*}$ follows. On the other hand, $\phi_{g}\left(\underline{X}_{g}\right)=\theta_{g}-\underline{X}_{g}$ and $\phi_{g}\left(\bar{X}_{g}\right)=\overline{X_{g}}-\theta_{g}$, so that $\phi_{g}\left(\underline{X}_{g}\right)>\phi_{g}\left(\bar{X}_{g}\right) \Rightarrow \theta_{g}>\left(\bar{X}_{g}+\underline{X}_{g}\right) / 2$. Symmetrically, suppose that $\phi_{g}\left(\underline{X}_{g}\right)=\phi_{g^{\prime}}\left(\underline{X}_{g^{\prime}}\right)<\phi_{g}\left(\bar{X}_{g}\right)=\phi_{g^{\prime}}\left(\bar{X}_{g^{\prime}}\right)$ (so that $\theta_{g}<\left(\bar{X}_{g}+\underline{X}_{g}\right) / 2$ ). Then, again, we have $\phi_{g}(x)>\phi_{g^{\prime}}(x)$ for all $x$, so that $x_{g}^{*}<x_{g^{\prime}}^{*}$

Proof of Proposition 2 For left truncations, this reduces to showing that, for all $x \in$ $\left(k, \bar{X}_{g}\right), \phi_{g}(x)-\phi_{g^{\prime}}(x)>0$. Note that

$$
\begin{aligned}
\phi_{g}(x)-\phi_{g^{\prime}}(x) & =E(X \mid X>x)-E(X \mid X<x)-[E(X \mid X>x)-E(X \mid X \in(k, x))] \\
& =E(X \mid X \in(k, x))-E\left(X \mid X \in\left(\underline{X}_{g}, x\right)\right)>0
\end{aligned}
$$

For right truncations, we have instead,

$$
\begin{aligned}
\phi_{g}(x)-\phi_{g^{\prime}}(x) & =E(X \mid X>x)-E(X \mid X<x)-[E(X \mid X \in(x, k))-E(X \mid X<x] \\
& =E\left(X \mid X \in\left(x, \bar{X}_{g}\right)\right)-E(X \mid X \in(x, k))>0
\end{aligned}
$$

for all $x \in\left(\underline{X}_{g}, k\right)$. Consider now a left censoring. This involves reassigning to point $k$ all the probability mass to the left of $k$. For all $x \in\left(k, \bar{X}_{g}\right)$,

$$
\begin{aligned}
\phi_{g}(x)-\phi_{g^{\prime}}(x) & = \\
E(X \mid X>x)-E(X \mid X<x)-\left[E(X \mid X>x)-\frac{F(k)}{F(x)} k-\frac{F(x)-F(k)}{F(x)} E(X \mid X \in(k, x))\right] & = \\
\frac{F(k)}{F(x)}\left[k-E\left(X \mid X \in\left(\underline{X}_{g}, k\right)\right)\right. & >0
\end{aligned}
$$

An analogous result is obtained in the symmetric case of right censoring. The proof is then concluded by noticing that the RHS of (3) is decreasing in the cutoff.

Proof of Lemma 4 We know that $f_{g}-f_{g^{\prime}}$ crosses zero at most three times. Hence, $F_{g}-F_{g^{\prime}}$ has at most three changes in monotonicity. Let $\underline{x} \equiv \min \left\{\underline{X}_{g}, \underline{X}_{g^{\prime}}\right\}$ and $\bar{x} \equiv \max \left\{\bar{X}_{g}, \bar{X}_{g^{\prime}}\right\}$. 
Clearly enough, $F_{g}(\underline{x})-F_{g^{\prime}}(\underline{x})=F_{g}(\bar{x})-F_{g^{\prime}}(\bar{x})=0$. Hence, the difference $F_{g}-F_{g^{\prime}}$ crosses zero at most twice for $x \in(\underline{x}, \bar{x})$. Since $f_{g}-f_{g^{\prime}}$ must be negative for $x$ small enough, $F_{g}-F_{g^{\prime}}$ must be initially negative. This implies that also the difference in the quantile functions $F_{g}^{-1}-F_{g^{\prime}}^{-1}$ must cross zero at most twice, and must be initially positive. Integrating again, we obtain that the difference in the Lorenz curves $\mathcal{L}_{g}(p)-\mathcal{L}_{g^{\prime}}(p)$ changes monotonicity at most twice. However, notice that $\mathcal{L}_{g}(0)-\mathcal{L}_{g^{\prime}}(0)=0$ and, since the two distributions have the same mean, $\mathcal{L}_{g}(1)-\mathcal{L}_{g^{\prime}}(1)=0$. Hence, once again, the difference $\mathcal{L}_{g}(p)-\mathcal{L}_{g^{\prime}}(p)$ must cross zero at most once, and must be initially positive.

Proof of Lemma 5 We know from Lemma 4 that either one distribution is second order stochastically dominated or the Lorenz curves must cross exactly once, with $\mathcal{L}_{g}(p)$ initially above $\mathcal{L}_{g^{\prime}}(p)$. Hence, in the latter case, there must exist $\hat{p}$ such that $\mathcal{L}_{g}(p)>\mathcal{L}_{g^{\prime}}(p)$ for all $p<\hat{p}$ and $\mathcal{L}_{g}(p)<\mathcal{L}_{g^{\prime}}(p)$ otherwise. From (7), this implies that $\Phi_{g}(p)>\Phi_{g^{\prime}}(p)$ for all $p<\hat{p}$ and $\Phi_{g}(p)<\Phi_{g^{\prime}}(p)$ for all $p>\hat{p}$.

Proof of Proposition 4 Consider then the case where neither distribution is second order dominated. Then, from Lemma 3 , there exists $\hat{p} \in(0,1)$ such that $\Phi_{g}(p)<\Phi_{g^{\prime}}(p)$ for all $p<\hat{p}$ and $\Phi_{g}(p)>\Phi_{g^{\prime}}(p)$ for all $p>\hat{p}$. Let $\hat{x}_{g} \equiv F_{g}^{-1}(\hat{p})$ and $\hat{x}_{g^{\prime}} \equiv F_{g^{\prime}}^{-1}(\hat{p})$. Clearly enough, $\phi_{g}(x)<\phi_{g^{\prime}}(x)$ for all $x<\min \left\{\hat{x}_{g}, \hat{x}_{g^{\prime}}\right\}$. Since $v(x)$ is increasing, if the proportion of abstainers $p$ is less than $\hat{p}$ in both groups, it must be that $x_{g}^{*}>x_{g^{\prime}}^{*}$. The reverse clearly happens if $x>\max \left\{\hat{x}_{g}, \hat{x}_{g^{\prime}}\right\}$, so that $p>\hat{p}$ in both groups.

Proof of Proposition 5 If $f_{g}$ and $f_{g^{\prime}}$ cross twice, then the CDFs $F_{g}$ and $F_{g^{\prime}}$ cross only once in the same interval. Hence, there exists $\hat{x} \in\left(\underline{X}_{g^{\prime}}, \bar{X}_{g^{\prime}}\right)$ such that $F_{g}(\hat{x})=F_{g^{\prime}}(\hat{x})$. This obviously implies that the quantile functions also cross once at $\hat{p} \equiv F_{g}(\hat{x})=F_{g^{\prime}}(\hat{x})$. From second order dominance, it must be that $F_{g}(x)$ crosses $F_{g^{\prime}}(x)$ initially from above. Hence, $F_{g}(x)<F_{g^{\prime}}(x)$ for all $x>\hat{x} \Leftrightarrow p>\hat{p}$. Since $x_{g^{\prime}}^{*}>x_{g}^{*}$ from Proposition 3 , then, if $x_{g}^{*} \geq \hat{x}, F_{g^{\prime}}\left(x_{g^{\prime}}^{*}\right)>F_{g}\left(x_{g}^{*}\right)$, which in turn implies $1-F_{g^{\prime}}\left(x_{g^{\prime}}^{*}\right)<1-F_{g}\left(x_{g}^{*}\right)$. In contrast, $p \leq \hat{p} \Leftrightarrow x \leq \hat{x} \Leftrightarrow F_{g}(x) \geq F_{g^{\prime}}(x)$. Hence, whether group $g^{\prime}$ or $g$ has more provision depends on how much larger $x_{g^{\prime}}^{*}$ is relative to $x_{g}^{*}$. In the limit, as $v(x)$ approaches a vertical line, then $x_{g^{\prime}}^{*} \rightarrow x_{g}^{*}$, which implies $1-F_{g^{\prime}}\left(x_{g^{\prime}}^{*}\right)>1-F_{g}\left(x_{g}^{*}\right)$.

Proof of Proposition 6 Equilibrium requires $\phi_{x}\left(x^{*}\right)=-v\left(x^{*}\right)$ or, for $z^{*} \equiv\left(x^{*}-\theta\right) / \sigma$,

$$
\phi_{x}\left(\theta+\sigma z^{*}\right) \equiv-v\left(\theta+\sigma z^{*}\right)
$$


Note that,

$$
\phi_{x}\left(x^{*}\right)=E\left[x \mid x>x^{*}\right]-E\left[x \mid x<x^{*}\right]=\sigma\left(E\left[z \mid z>z^{*}\right]-E\left[z \mid z<z^{*}\right]\right) \equiv \sigma \phi_{z}\left(z^{*}\right),
$$

and that, different from $\phi_{x}\left(x^{*}\right), \phi_{z}\left(z^{*}\right)$ depends on $\sigma$ only through the cutoff. Equation 22 thus becomes

$$
\sigma \phi_{z}\left(z^{*}\right) \equiv-v\left(\theta+\sigma z^{*}\right)
$$

Differentiating both sides of the identity with respect to $\sigma$, we obtain

$$
\frac{d z^{*}}{d \sigma}=-\frac{v^{\prime}\left(\theta+\sigma z^{*}\right) z^{*}+\phi_{z}\left(z^{*}\right)}{\sigma\left(v^{\prime}\left(\theta+\sigma z^{*}\right)+\phi_{z}^{\prime}\left(z^{*}\right)\right)}
$$

Note that the denominator is always positive in any stable equilibrium (this is because $-v(x)$ must cross $\phi(x)$ from above, so that $-v^{\prime}<\phi^{\prime}-$ whenever $-v(x)$ crosses from below, the fixed point is knife edge). Since $v^{\prime}>0$, the term $v^{\prime} z^{*}+\phi_{z}$ is also positive for all $z^{*} \geq 0$ (i.e. $x^{*} \geq \theta$ ). Consider first $v(\theta)<0$. We want to establish conditions under which $d z^{*} / d \sigma$ is negative. This is clearly the case when $z^{*} \geq 0$. Assume then $z^{*}<0$ (or $x^{*}<\theta$ ). Using the equilibrium identity (24) to replace $\phi_{z}\left(z^{*}\right)$, the term $v^{\prime} z^{*}+\phi_{z}$ has the same sign as

$$
v^{\prime}\left(\theta+\sigma z^{*}\right) \sigma z^{*}-v\left(\theta+\sigma z^{*}\right)
$$

or, equivalently,

$$
v^{\prime}\left(x^{*}\right)\left(x^{*}-\theta\right)-v\left(x^{*}\right)
$$

which is equal to

$$
\left[v(\theta)-v\left(x^{*}\right)-\left(v^{\prime}\left(x^{*}\right) \theta-v^{\prime}\left(x^{*}\right) x^{*}\right)\right]-v(\theta)
$$

Note that, given $v($.$) increasing, the term in brackets is non-negative (non-positive) for all$ $x^{*}<\theta$ when $v($.$) is weakly convex (concave). Given v(\theta)<0$, there exists $\epsilon>0$ such that if $v^{\prime \prime}(x)>-\epsilon$ for all $x$, expression (28) is positive, which implies $d z^{*} / d \sigma<0$. Consider now $v(\theta)>0$. Clearly, there exists $\epsilon>0$ such that, if $v^{\prime \prime}(x)<\epsilon$ for all $x$, expression (28) is negative for all $x^{*}<\theta$. Moreover, given $v(\theta)>0$, all types $x \geq \theta$ strictly prefer to provide the signal, so that, necessarily, $x^{*}<\theta\left(z^{*}<0\right)$. This implies $d z^{*} / d \sigma>0$. Consider then $\sigma^{\prime}$ and $\sigma^{\prime \prime}>\sigma^{\prime}$. Denoting with $H$ the CDF of the standardized variable $z$, it follows that, if $v(\theta)<0$ and $v^{\prime \prime}(x)>-\epsilon$ for all $x, F_{\sigma^{\prime}}\left(x^{*}\left(\sigma^{\prime}\right)\right)=H\left(z^{*}\left(\sigma^{\prime}\right)\right)>H\left(z^{*}\left(\sigma^{\prime \prime}\right)\right)=F_{\sigma^{\prime \prime}}\left(x^{*}\left(\sigma^{\prime \prime}\right)\right)$, so that aggregate provision $1-F_{\sigma}\left(x^{*}\right)$ is larger when $\sigma=\sigma^{\prime \prime}$. The reverse applies when $v($.$) is$ not too convex and $v(\theta)>0$. 
Proof of Proposition 8 We give the proof for PBE where, for each action $a \in\{0,1\}$, there exists at least one group where $a$ is played by a positive measure of agents, but the argument trivially extends to PBE where the same action is played in all groups. Fix an equilibrium group structure $S$. Given $S$, there must exist, for all $a \in\{0,1\}$ at least one group $g_{a}$ with a positive fraction of members choosing action $a$. Moreover, in any equilibrium, an individual choosing $a$ must be indifferent between joining $g_{a}$ and any other group with a positive fraction of members who choose $a$ (and must weakly prefer these groups to any other group). This implies that all type $x$ individuals choose $a=1$ iff $E\left(x \mid a=1, g_{1}\right)-E\left(x \mid a=0, g_{0}\right) \geq-v(x)$ irrespectively of their group membership. Hence, given monotonicity of $v$, there exists a $\hat{x}$ such that $a=1 \Leftrightarrow x \geq \hat{x}$ irrespectively of group membership. Let $y_{a}(g)$ denote the measure of agents choosing action $a$ which is in group $g$. From the law of iterated expectations $\sum_{g=1}^{N} y_{1}(g) E(x \mid a=1, g)=E_{G}(x \mid x \geq \hat{x})$ and $\sum_{g=1}^{N} y_{0}(g) E(x \mid a=0, g)=E_{G}(x \mid x<\hat{x})$ where the $G$ subscript is used to stress that the expectation is taken under the aggregate density $f_{G}$. However, since $E(x \mid a, g)=E\left(x \mid a, g_{a}\right)$ for all groups such that $y_{a}(g)>0$, $E\left(x \mid a=0, g_{0}\right)=E_{G}(x \mid x<\hat{x})$ and $E\left(x \mid a=1, g_{1}\right)=E_{G}(x \mid x \geq \hat{x})$ follow. Equilibrium then implies that $\hat{x}$ must satisfy $\phi_{G}(\hat{x})=-v(\hat{x})$. This in turn implies that $\hat{x}$ must be an equilibrium cutoff under $S_{G}$.

\section{References}

[1] Adriani, F. and Sonderegger, S. (2009). Why do parents socialize their children to behave pro-socially? An information-based theory. Journal of Public Economics, 93: 1119-1124.

[2] Adriani, F. and Sonderegger, S. (2018). Signaling about norms: Socialization under strategic uncertainty. Scandinavian Journal of Economics, 120: 685-716.

[3] Adriani F., Matheson J.A., Sonderegger S. (2018) Teaching by example and induced beliefs in a model of cultural transmission. Journal of Economic Behavior and Organization, 145:511-29.

[4] Andreoni, J., and Bernheim, B.D. (2009). Social image and the 50-50 norm: A theoretical and experimental analysis of audience effects. Econometrica, 77: 1607-1636. 
[5] Arnott, R., and Rowse, J. (1987). Peer group effects and educational attainment. Journal of Public Economics, 32: 287-305.

[6] Atkinson, A. (1970). On the measurement of inequality. Journal of Economic Theory, 2: $244-263$.

[7] Banerjee, A.V., and Besley, T. (1991) Peer group externalities and learning incentives: A theory of nerd behavior. Mimeo.

[8] Barron, J.M., and Gjerde, K.P. (1997). Peer pressure in an agency relationship. Journal of Labor economics, 15: 234-254.

[9] Bénabou, R. (1993). Workings of a city: Location, education, and production. The Quarterly Journal of Economics, 108: 619-652.

[10] Bénabou, R., and Tirole, J. (2006). Incentives and prosocial behavior, American Economic Review, 96: 1652-1678.

[11] Bénabou, R., and Tirole, J. (2011). Laws and norms, National Bureau of Economic Research.

[12] Bernheim, B. D. (1994). A theory of conformity. Journal of political Economy, 102: 841-877.

[13] Bisin, A. and Verdier, T. (2001). The economics of cultural transmission and the dynamics of preferences. Journal of Economic theory, 97: 298-319.

[14] Bowles, S., and Park, Y. (2005). Emulation, inequality, and work hours: Was Thorsten Veblen right? Economic Journal, 115: 397-412.

[15] Charles, K.K., Hurst, E., and Roussanov, N. (2009). Conspicuous consumption and race. Quarterly journal of economics, 124: 425-467.

[16] Corneo, G., and Grüner, H. P. (2000). Social limits to redistribution. American Economic Review, 90: 1491-1507.

[17] Dubey, P. and Geanakoplos, J. (2010). Grading exams: 100, 99, 98,... or A, B, C? Games and Economic Behavior, 69: 72-94. 
[18] Ellingsen, T., and Johannesson, M. (2007). Paying Respect. Journal of Economic Perspectives, 21: 135-149.

[19] Ellingsen, T., and Johannesson, M. (2008). Pride and prejudice: The human side of incentive theory. American Economic Review, 98(3), 990-1008.

[20] Frank, R. H. (1985a). Choosing the right pond: Human behavior and the quest for status. Oxford University Press. Chicago

[21] Frank, R. H. (1985b). The demand for unobservable and other nonpositional goods. The American Economic Review, 75: 101-116.

[22] Frank, R. H., Levine, A. S., and Dijk, O. (2005). Expenditure cascades. Cornell University mimeograph.

[23] Glazer, A., and Konrad, K.A. (1996). A signaling explanation for charity, American Economic Review, 86: 1019-28.

[24] Grout, P.A., Mitraille, S. and Sonderegger, S. (2015). The costs and benefits of coordinating with a different group. Journal of Economic Theory, 160: 517-535.

[25] Harbaugh, R. (2010). Prospect Theory or skill signaling? Mimeo.

[26] Harbaugh, R., and Rasmussen, E., (2014). Coarse grades, forthcoming in AEJ: Microeconomics.

[27] Harsanyi J.C. (1980) Essays on ethics, social behavior, and scientific explanation, D. Reidel Publishing Company, Dordrecht, Holland.

[28] Heffetz, O., and Frank, R.H. (2008). Preferences for status: Evidence and economic implications. Handbook of social economics, 1, 69-91.

[29] Hopkins, E., and Kornienko, T. (2004) Running to Keep in the Same Place: Consumer Choice as a Game of Status. American Economic Review, 94: 1085-1107.

[30] Hopkins, E., and Kornienko, T. (2009) Status, Affluence, and inequality: Rank-based comparisons in games of status. Games and Economic Behavior, 67: 552-568. 
[31] Hopkins, E. and Kornienko, T. (2010). Which inequality? The inequality of endowments versus the inequality of rewards. American Economic Journal: Microeconomics, 2: 10637.

[32] Ireland, N.J. (1994). On limiting the market for status signals. Journal of public Economics, 53: 91-110.

[33] Levy, G. and Razin, R. (2015). Preferences over equality in the presence of costly income sorting. American Economic Journal: Microeconomics, 7: 308-37.

[34] Jensen, M.K. (2018). Distributional comparative statics. Review of Economic Studies, 85: 581-610.

[35] Jewitt, I. (2004) Notes on the 'shapes' of distributions. Unpublished Manuscript.

[36] Kandel, E., and Lazear, E. P. (1992). Peer pressure and partnerships. Journal of political Economy, 100: 801-817.

[37] Lazear, E.P. (2001). Educational production. Quarterly Journal of Economics, 116: 777803.

[38] Lee, S. (2007). The timing of signaling: To study in high school or in college? International Economic Review, 48: 785-807.

[39] Marsh, H.W. (1987). The big-fish-little-pond effect on academic self-concept. Journal of educational psychology, 79: 280-295.

[40] Marsh, H.W., Chessor, D., Craven, R., and Roche, L. (1995). The effects of gifted and talented programs on academic self-concept: The big fish strikes again. American Educational Research Journal, 32: 285-319.

[41] Mazali, R., and Rodrigues-Neto, J.A. (2013) Dress to impress: Brands as status symbols. Games and Economic Behavior, 82:103-131.

[42] Milgrom, P. and Roberts, J., (1994). Comparing equilibria. American Economic Review, 84:441-459.

[43] Postlewaite, A. (1998) The social basis of interdependent preferences. European Economic Review, 42:779-800. 
[44] Postlewaite, A. (2011) Social norms and preferences. Handbook for Social Economics, J. Benhabib, A. Bisin and M. Jackson, eds., 31-67.

[45] Spence, M. (1973) Job market signaling. Quarterly Journal of Economics, 3:355-74.

[46] Weiss, Y., and Fershtman, C. (1998). Social status and economic performance:: A survey. European Economic Review, 42: 801-820.

[47] Zubrickas, R. (2015). Optimal grading. International Economic Review, 56: 751-776. 